\title{
N6-methyladenosine (m6A)-mediated messenger RNA signatures and the tumor immune microenvironment can predict the prognosis of hepatocellular carcinoma
}

\author{
Shen Shen ${ }^{1,2 \#}$, Jingya Yan ${ }^{1,2 \#}$, Yize Zhang ${ }^{1,2}$, Zihui Dong ${ }^{1,2}$, Jiyuan Xing ${ }^{1,2}$, Yuting He ${ }^{1}$ \\ ${ }^{1}$ Gene Hospital of Henan Province, Precision Medicine Center, The First Affiliated Hospital of Zhengzhou University, Zhengzhou, China; \\ ${ }^{2}$ Department of Infectious Diseases, the First Affiliated Hospital of Zhengzhou University, Zhengzhou, China \\ Contributions: (I) Conception and design: All authors; (II) Administrative support: Y He, S Shen; (III) Provision of study materials or patients: J Yan, Z \\ Dong; (IV) Collection and assembly of data: Y Zhang; (V) Data analysis and interpretation: S Shen, J Xing; (VI) Manuscript writing: All authors; (VII) \\ Final approval of manuscript: All authors. \\ \#These authors contributed equally to this work. \\ Correspondence to: Yuting He. Gene Hospital of Henan Province, Precision Medicine Center, The First Affiliated Hospital of Zhengzhou University, \\ No.1 Jianshe Road, Zhengzhou 450052, China. Email: fccheyt1@zzu.edu.cn.
}

Background: N6-methyladenosine (m6A)-mediated ribonucleic acid (RNA) methylation is considered to be the most significant and abundant epigenetic modification in eukaryotic cells, and plays an essential role in the carcinogenesis and molecular pathogenesis of hepatocellular carcinoma (HCC). However, the relationship between $\mathrm{m} 6 \mathrm{~A}$ regulation and immune cell infiltration of the tumor immune microenvironment (TIME) has not yet been clarified. We aimed to investigate the roles of m6A RNA gene regulators in HCC immune regulation and prognosis.

Methods: The Cancer Genome Atlas (TCGA) database was used, and unsupervised clustering of $21 \mathrm{~m} 6 \mathrm{~A}$ regulators was performed based on differential gene expression. Gene Set Variation Analysis (GSVA), singlesample Gene Set Enrichment Analysis (ssGSEA), the empirical Bayes method, and m6A scores were used in our analyses.

Results: Of 433 samples, 101 (23.22\%) had m6A regulatory factor mutations. From these, we identified three m6A subtypes, which correlated with different TIME phenotypes: immune rejection, immune infiltration, and immune deficiency. Tumors with low methyltransferase-like 3 (METTL3) expression had increased infiltration of dendritic cells (DCs) in the TIME. Reduced METTL3 expression also led to an overall increase in expression of major histocompatibility complex (MHC) molecules, costimulatory molecules, and adhesion molecules. The m6A subtypes were scored and analyzed for correlations. Patients with epithelial-mesenchymal transition (EMT) subtypes had lower m6A scores than the other three molecular subtypes. Survival analysis found that patients with low m6A scores had better overall survival [hazard ratio (HR) $1.6(1.1-2.3)$ ] and a 1.16 times better 5-year survival rate than patients with high m6A scores $(56 \%$ vs. $48 \%)$.

Conclusions: Our results demonstrated that three different m6A modification subtypes contribute to immune regulation in HCC and have potential as novel prognostic indicators and immune therapeutic targets.

Keywords: N6-methyladenosine (m6A); Hepatocellular carcinoma (HCC); tumor immune microenvironment (TIME); methyltransferase-like 3 (METTL3); survival

Submitted Oct 19, 2020. Accepted for publication Dec 08, 2020.

doi: $10.21037 / \mathrm{atm}-20-7396$

View this article at: http://dx.doi.org/10.21037/atm-20-7396 


\section{Introduction}

Hepatocellular carcinoma (HCC) remains a high incidence malignancy with high mortality rate and poor prognosis worldwide. It has been reported that there are approximately 850,000 new HCC patients annually, with about 500,000 deaths due to HCC-associated diseases $(1,2)$. Hepatitis B and $\mathrm{C}$ viral infections, excess alcohol consumption, aflatoxin B1 exposure, obesity, diabetes, and metabolic diseases are major risk factors for the development of HCC (3). Owing to the difficulty in making an early diagnosis, HCC patients are typically diagnosed at an advanced stage of the disease, and thus, treatments are generally associated with a low survival rate, high risk of recurrence, malignant metastasis, as well as a high risk of selection and spreading of drug resistance (4). Recently, immune therapies, such as immune checkpoint inhibition, have been used in advanced HCC to modify the carcinogenic process and to augment adaptive immunity (5). It has been shown that the tumor immune microenvironment (TIME), which is composed of both pro- and anti-tumor immune cells, can be reprogrammed by tumor-derived factors, which are involved in immune evasion and tumor progression $(6,7)$. Accumulating evidence supports a role for the TIME in determining HCC progression, recurrence, metastasis, and poor outcome $(8,9)$. Tumor-infiltrating immune cells (TIICs) are important components of the TIME and have been shown to be significant predictors of HCC survival (10). However, correlations between TIME parameters and HCC genesis and development are still poorly understood (10).

Currently, the most well studied messenger ribonucleic acid (mRNA) modification is N6-methyladenosine (m6A). It is the most pervasive internal mRNA modification (10), and correlates with tumor progression (11). Previous studies have shown that m6A demethylation of the fat mass and obesity-associated protein (FTO) is up-regulated and promotes tumor proliferation and metastasis in breast cancer $(12,13)$. However, the role of the m6A modification in HCC tumorigenesis remains unclear.

We hypothesized that $\mathrm{m} 6 \mathrm{~A}$ may regulate $\mathrm{HCC}$ tumorigenesis and progression by regulating the immune system and thus may be a therapeutic drug target for HCC. To evaluate this hypothesis, data from The Cancer Genome Atlas (TCGA) were used as an effective cohort, and data from the Gene Expression Omnibus (GEO) database were used as a validation cohort. Genes associated with the immune microenvironment were downloaded from the Immunology Analysis Portal database (https//www. immport.org/shared/genelists), analyzed, and immune phenotypes were classified by immune scores. We validated the different m6A modification patterns and explored the relationship between immune infiltration and $\mathrm{m} 6 \mathrm{~A}$ regulation. We also identified three different immune phenotypes: the immune rejection phenotype, the immune inflammation phenotype, and the immune desert phenotype, and found that formation of the tumor microenvironment plays a role in the phenotype. We combined m6A modification patterns and immune infiltration phenotypes to establish a system to estimate HCC prognosis.

We present the following article in accordance with the REMARK reporting checklist (available at http://dx.doi. org/10.21037/atm-20-7396).

\section{Methods}

\section{Data collection}

The work-flow of this study is illustrated in Figure S1A. All related information was downloaded from TCGA data portal (http://cancergenome.nih.gov/). And the study was conducted in accordance with the Declaration of Helsinki (as revised in 2013). Data cohorts with missing information were removed. Data collection, refinement, and model statistics are summarized in Table S1. RNA sequence data from the National Cancer Institute's (NCIs) Genomic Data Commons (GDC, https://gdc.cancer.gov/about-data/ publications/pancanatlas), Fragments Per Kilobase per total Million mapped reads (FPKM), and TCGA biolinks were also used $(14,15)$. Data were analyzed using the statistical software package R, version 3.6.1. Raw data were processed into R/Bioconductor.

\section{Unsupervised gene cluster analysis}

We used clustering, a resampling-based clustering algorithm, to analyze the m6A gene profiles of HCCs. A total of $21 \mathrm{~m} 6 \mathrm{~A}$ regulators, including eight writers, two erasers, and 11 readers were analyzed. We performed unsupervised cluster analysis with a consistent clustering algorithm and the Consensus Cluster Plus package as described previously (16).

\section{Gene Set Variation Analysis (GSVA) and functional annotation}

For an in-depth analysis of the biological processes that 
associate with the different m6A modification patterns, the "GSVA" R package for GSVA enrichment analysis was utilized. The gene set "c2.cp.kegg. v6.2. symbols" was downloaded from the MSigDB database and used to run GSVA analysis. For functional annotation of genes, we utilized cluster analysis and a false discovery rate $<0.05$.

\section{Estimation of the immune cells infiltrating the TIME}

A gene set of a variety of human immune cell subtypes was obtained from Charoentong et al. (17). Single-sample Gene Set Enrichment Analysis (ssGSEA) calculates separate enrichment scores for each sample and gene pair. This analysis showed the relative abundance of each cell type that infiltrated the TIME for each sample.

\section{Classification of m6A phenotypes based on differential gene expression}

To validate and classify m6A-associated genes, differentially expressed gene (DEG) analysis (fold-changes) and statistical computations of the two conditions were conducted using the EBSeq R package (https://bioconductor.org/packages/ release/bioc/html/EBSeq.html).

\section{Construction of m6A gene markers and m6A scoring}

To validate m6A regulator modifications in HCC samples, we established a scoring system to estimate $m 6 \mathrm{~A}$ gene characteristics. To establish $m 6 A$ gene markers, DEGs of the different m6A clusters were normalized to extract overlapping genes, the unsupervised clustering method was used to analyze the overlapping data, and patients were divided into multiple groups for in-depth analysis. The number and stability of gene clusters were defined by the consistent clustering algorithm. We then used a univariate Cox regression model to analyze the association of each gene cluster with prognosis. We selected principal component 1 and principal component 2 as signature scores. Subsequently, we defined the m6a score using a method like that used in analyzing gene-gene interactions (GGIs): $m 6 \mathrm{~A}$ Score $=\Sigma(P C 1 i+P C 2 i)$.

\section{Analysis of m6A modifications and their immune-related signatures in $\mathrm{HCC}$}

To investigate associations between m6A modifications and the immune microenvironment, we downloaded immune- related genes from the ImmPort database (http://www. immport.org), and identified prognostic gene signatures based on genes with independent prognostic values as determined previously (18). Our m6A scoring system revealed that $\mathrm{m} 6 \mathrm{~A}$ gene characteristics are associated with particular biological pathways.

\section{Statistical analysis}

DEGs were applied in this study with the threshold of absolute $\log 2$-fold-change $>1$ and an adjusted $\mathrm{P}$ value $<0.05$ using the R package "limma". The overall survival rate was evaluated using the log-rank test with Kaplan-Meier estimation. Differences between the groups were evaluated by analysis of variance (ANOVA). All $\mathrm{P}$ values were twosided, and $\mathrm{P}$ values $<0.05$ were considered significant. Comparative studies were analyzed using the Kruskal-Wallis test and Wilcoxon test. ${ }^{*} \mathrm{P}<0.05,{ }^{* *} \mathrm{P}<0.01,{ }^{* * *} \mathrm{P}<0.001$. All statistics were calculated using $\mathrm{R}$ language.

\section{Results}

\section{Expression of $m 6 A$ regulators in $\mathrm{HCC}$}

To better understand the search strategy and selection of datasets for comprehensively understand $\mathrm{m} 6 \mathrm{~A}$ regulation in HCC progression, we constituted a flow chart of this study (Figure S1A). In total, 371 patients were involved in this study and three clusters were identified. The transcription levels of $21 \mathrm{~m} 6 \mathrm{~A}$ regulators, which included 11 readers, eight writers, and two erasers, as well as the copy number variations (CNVs) and somatic mutations of these regulators, were described in a previous study (19). The chromosomal locations of the amplification mutations were distributed randomly and unequally across the chromosomes (Figure 1A). Of 64 HCC samples, 44 contained mutations related to $\mathrm{m} 6 \mathrm{~A}$ modifications (68.75\% mutation rate). The most prevalent mutation were found in the KIAA1429 (8\%) and LRPPRC (8\%) genes, followed by HNRNPC (6\%) and YTHDC2 (6\%) (Figure 1B). The $21 \mathrm{~m} 6 \mathrm{~A}$ regulators exhibited widespread gene gain and loss mutations, KIAA1429 and YTHDF3 contained the most gain mutation type, while $A L K B H 5$ and $\mathrm{ZC} 3 \mathrm{H} 13$ had the most frequent loss mutation type (Figure 1C). We also explored the 21 regulators in tumor tissue compared with normal tissue, and easily identified the two subgroups; the red spot represented normal tissue and the blue spot represented tumor tissue (Figure 1D). 
A

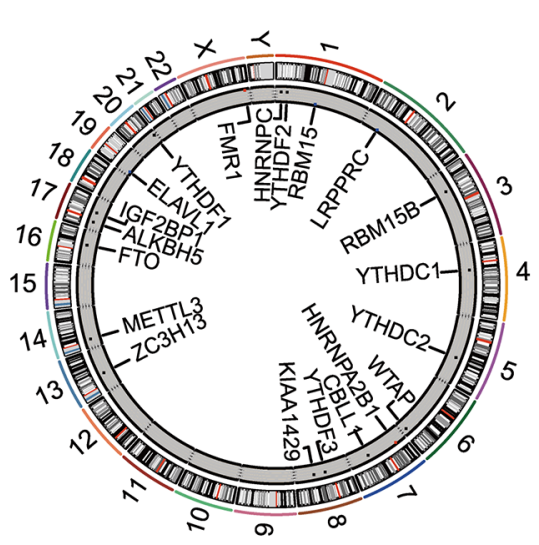

C



B

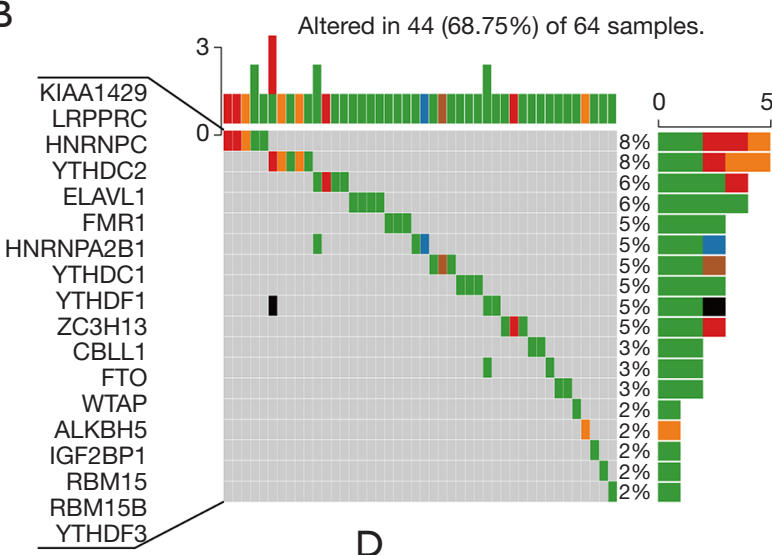

Missense mutation

- Nonsense mutation

- Splice Site

- In Frame Del

- Frame Shift Del

- Multi Hit

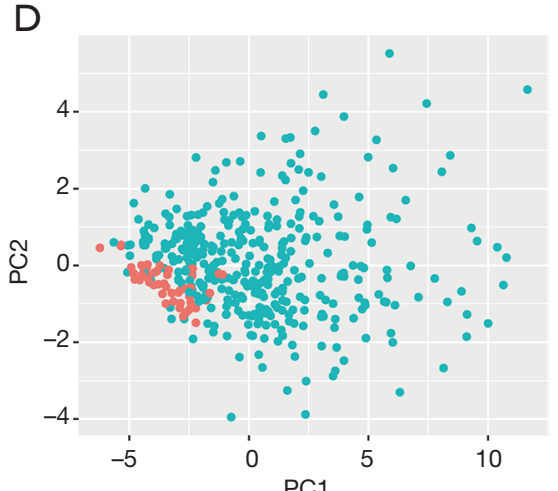

Factor (coltype)

- Normal

- Tumor

$\mathrm{E}$

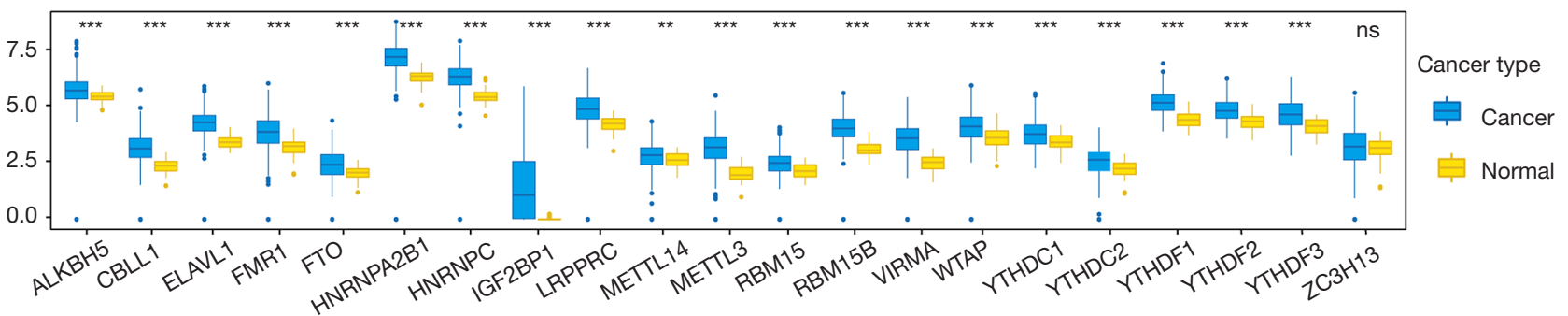

Figure 1 Overview of m6A gene locus and gene information. (A) The m6A regulators mutation location. (B) Waterfall plot of m6A regulators mutation gene and mutation type. (C) Frequency plots of copy number gains (in green) and losses (in red) defined in all m6A regulators. (D) Regulators could be divided into two subgroups (red and green) based on the gene expression level. (E) Comparison of gene expression level of 21 regulators between the normal and tumor tissue cohort. ${ }^{* *} \mathrm{P}<0.01,{ }^{* * *} \mathrm{P}<0.001$. m6A, N6-methyladenosine.

To further investigate the overall survival between the regulators, HCC patients were divided into mutated subgroup and wide type subgroup based on whether they were combined with m6A mutation information. Obviously, the mutated subgroups had a poor prognosis in $Y T H D F 1$ $[\mathrm{P}=0.018$, hazard ratio $(\mathrm{HR})=4.7], \operatorname{LRPPRC}(\mathrm{P}=0.003$, $\mathrm{HR}=6.44)$, and FTO $(\mathrm{P}=4.12 \mathrm{e} 7, \mathrm{HR}=11.3)$ (Figure $\mathrm{S} 1 \mathrm{~B})$. We also performed a meta-analysis, and the results revealed that the regulators have prognostic potential in HCC cohorts (Figure $\mathrm{S1C}$ ). The mean expression levels of $\mathrm{m} 6 \mathrm{~A}$ regulators in HCC specimens and normal tissues are shown in
Figure $1 E$; the difference was significant and $\mathrm{m} 6 \mathrm{~A}$ regulators were highly expressed in both tumor tissue and normal tissue. In this study, we mapped the gene somatic mutation information of $21 \mathrm{~m} 6 \mathrm{~A}$ regulators between tumor tissue and normal tissue, and confirmed that m6A modification plays an important role in HCC tumorigenesis and progression.

\section{$m 6 A$ regulators have close relationship with cellular crucial functional pathways regulation}

In order to comprehensively explain the crosslink among 
the $21 \mathrm{~m} 6 \mathrm{~A}$ regulators, we divided $21 \mathrm{~m} 6 \mathrm{~A}$ regulators into three clusters as illustrated in Figure $2 A$. Through GSVA of functional genes, we determined the biological roles of the $\mathrm{m} 6 \mathrm{~A}$ regulators. The expression of genetic information of the $\mathrm{m} 6 \mathrm{~A}$ regulators was controlled through intricate regulatory networks and a complex regulatory relationship. The overall survival curve implied a significant survival advantage for cluster $2\left({ }^{*} \mathrm{P}<0.0001\right.$, Figure $\left.2 B\right)$.

In the present study, we explored the relationship between different modification patterns and biological functional activities. The results indicated that three clusters exhibit significant pathway enrichment. The cluster 1 -associated pathways gather on translation functions, such as olfactory transduction, neuroactive ligand receptor interaction, arachidonic acid metabolism complements and coagulation cascades, folate biosynthesis, arginine and proline metabolism, metabolism of xenobiotics by cytochrome, glutathione metabolism and Parkinson's disease cardiac muscle contraction, amyotrophic lateral sclerosis, prion diseases, and autoimmune diseases. Cluster 2 functional pathways were significantly enriched in the ERBB signaling pathway, cell cycle signaling pathway, cell adhesion, as well as other pathways related to cell matrix adhesion and carcinogenesis, such as colorectal cancer, chronic myeloid leukemia, adherent's junction, the ERBB signaling pathway, endometrial cancer, the cell cycle, oocyte meiosis, RNA degeneration, ubiquitin mediated proteolysis, basal transcription factors, non-homologous end joining, and thyroid cancer (Figure 2C). The third clusterassociated functional pathways act in metabolism pathways (Figure $2 D$ ). Our results implied that $\mathrm{m} 6 \mathrm{~A}$ regulators participated in many important cellular activities, including cellular metabolism regulation, nuclear factor regulation, signal transitional regulation, and carcinogenesis.

\section{$m 6 A$ regulators have a close relationship with the infiltration of immune cells}

Next, we estimated the enrichment score of immune cells among the three clusters and identified the differences in the infiltration of immune cells among clusters 1-3. The box plot exhibited significant differences in most immune cells, including activated B cells, activated cluster of differentiation (CD)4+T cells, activated CD8+T cells, ${ }^{\gamma \delta} \mathrm{T}$ cells, regulatory $\mathrm{T}$ cells, type 1 helper cells, type 17 helper cells, activated dendritic cells (DCs), CD56 bright natural killer (NK) cells, CD56 dim NK cells, eosinophils, macrophages, and mast cells (Figure $3 A$ ).
To further investigate the biological behaviors among the three m6A modification clusters, pathway enrichment analysis was applied. Our results indicated that the transforming growth factor beta (TGF- $\beta$ ) pathway, angiogenesis, and the tumor EMT process were remarkably different among the clusters (Figure 3B). Our research suggested that the three types of regulators were involved in crucial functional regulations with different pathways. According to the GSVA analysis, the $21 \mathrm{~m} 6 \mathrm{~A}$ regulators were classified as either an immune rejected phenotype, an immune inflamed phenotype, or an immune desert phenotype based on the infiltration of immune cells (Figure $3 A, B)$.

We then focused on methyltransferase like 3 (METTL3), and the immune score of different METTL3 expression subgroups showed a weak difference (Figure 3C). Furthermore, Spearman correlation analysis was performed for TIME infiltration and the m6A regulators (Figure 3D). METTL3 expression correlated with many of the TIME infiltrating immune cells and the immune score. Our study implied that the infiltration of CD4+ T cells, type 1 T helper cells, type 2 T helpers, CD56 bright NK cells, eosinophils, macrophages, mast cells, monocytes, and neutrophils exhibited a significant difference between high and low METTL3 expression (Figure S2A), indicating that low METTL3 expression resulted in increased TIMEassociated immune cell infiltration.

Subsequently, we estimated the gene expression of immune checkpoint molecules and receptors, and discovered that CD80, CD86, intercellular adhesion molecule (ICAM) 1, ICAM3, and programmed cell death ligand 1 (PDL1) gene expression showed differences across different METTL3 expression levels (Figure S2B). We then evaluated the enrichment score of some crucial cell activity pathways between two clusters, and the box plot exhibited significant differences in the TGF- $\beta$ pathway, $\mathrm{T}$ cell activation, angiogenesis, and the tumor EMT process (Figure S2C). These results indicated that the m6A regulators play key roles in immune cell infiltration and characteristic TIME formation.

\section{The transcriptome and clinical features of $m 6 A$ regulators}

To further identify the cellular biological behavior and indepth mechanisms of the m6A regulators, we performed unsupervised cluster analysis on m6A phenotyperelated genes to divide patients into one of four different clusters based on the m6A modified genomic phenotypes 
A

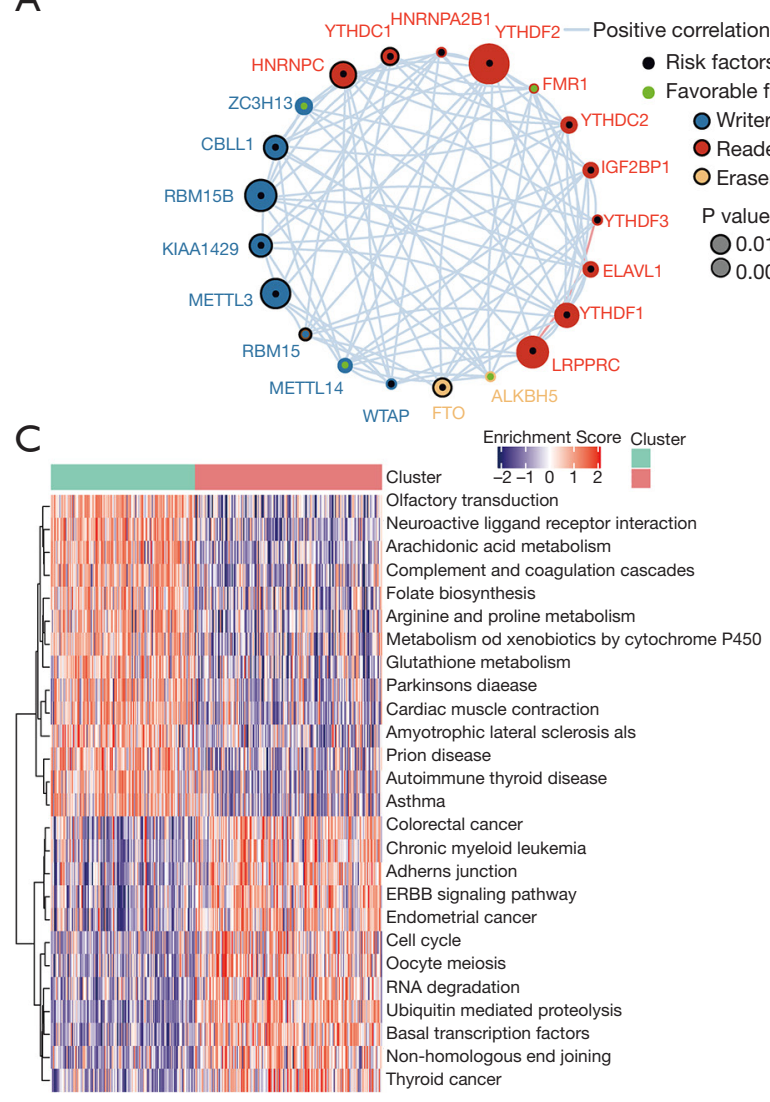

B

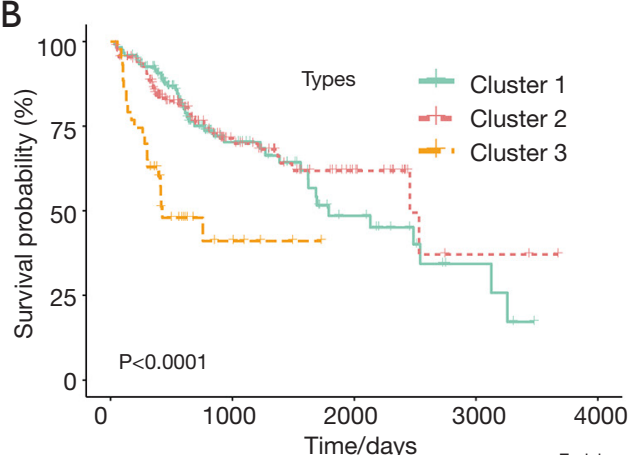

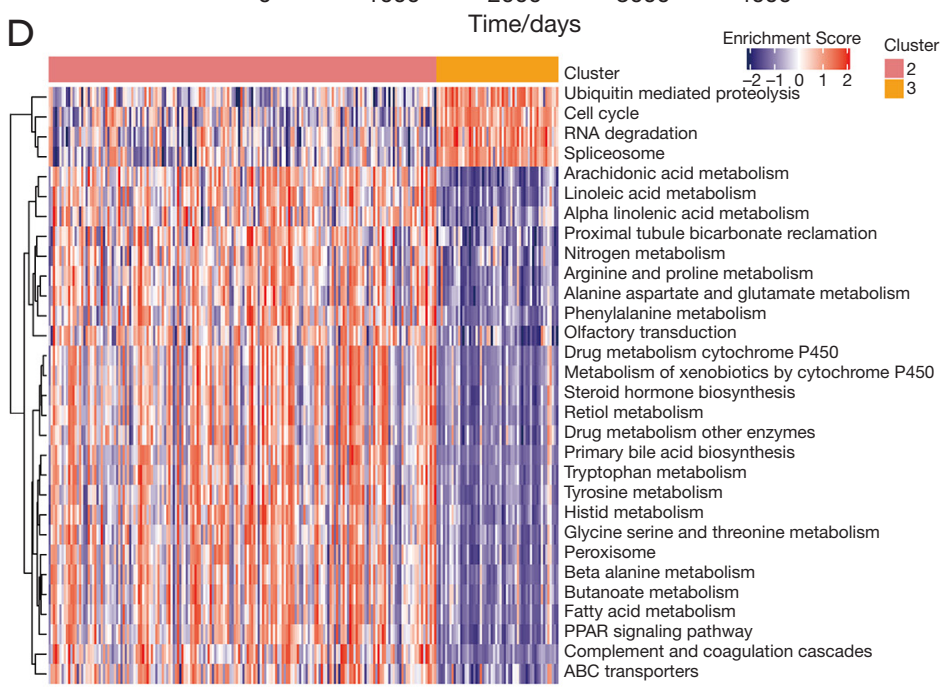

Figure 2 Crosslinks between $21 \mathrm{~m} 6 \mathrm{~A}$ regulators and associated biological activities. (A) Landscape and inner crosslink between $21 \mathrm{~m} 6 \mathrm{~A}$ regulators. (B) Kaplan-Meier curve of survival probability between three clusters. (C) Cluster enrichment analysis of biological activities between cluster 1 and cluster 2. (D) Cluster enrichment analysis of biological activities between cluster 2 and cluster 3. m6A, N6methyladenosine.

(Figure $4 A$ ). Based on the m6A regulator classifications, we confirmed that cluster 1 , with the abundant immune cell infiltration subgroup, exhibited a better prognosis and survival probability (Figure 4B). Among the three immune cell infiltration-associated subgroups, expression of regulatory factors of 21 regulators differed significantly (Figure 4C).

Further analysis of the matrix-related pathways revealed that the gene expression level of the m6A regulators suggested significantly different expression levels of immune related functional annotation and activation of matrix pathways, including CD8+T cells effector, angiogenesis, the cell cycle, cell cycle regulator, deoxyribonucleic acid (DNA) damage repair, DNA replication, EMT1, Fanconi anemia, homologous recombination, mismatch repair, nucleotide excision repair, and Pan-F-TBRS (Figure 4D). Here, we confirmed that the 21 regulators were classified into three gene clusters based on gene expression and cluster analysis. The box plots illustrated that the 21 regulators gene expression clusters were consistent with the functional classification.

\section{The m6A regulators gene expression classification with immune infiltration}

To better annotate the functions of m6A-related genes with immune cells, our analysis results implied that the infiltration of activated CD4+ $\mathrm{T}$ cells, regulatory $\mathrm{T}$ cells, type $17 \mathrm{~T}$ helper cells, CD56 bright NK cells, CD56 dim NK cells, and eosinophils exhibited significant differences between the three clusters (Figure 5A). To explore the roles of m6A-related phenotypes in the modulation of the TIME, we analyzed the expression of chemokines and cytokines in the three m6A gene clusters. Interferon gamma $(I F N-\gamma), C D 8 A$, T-Box transcription factor 2 (TBX2), and tumor necrosis factor (TNF) showed significant differences 

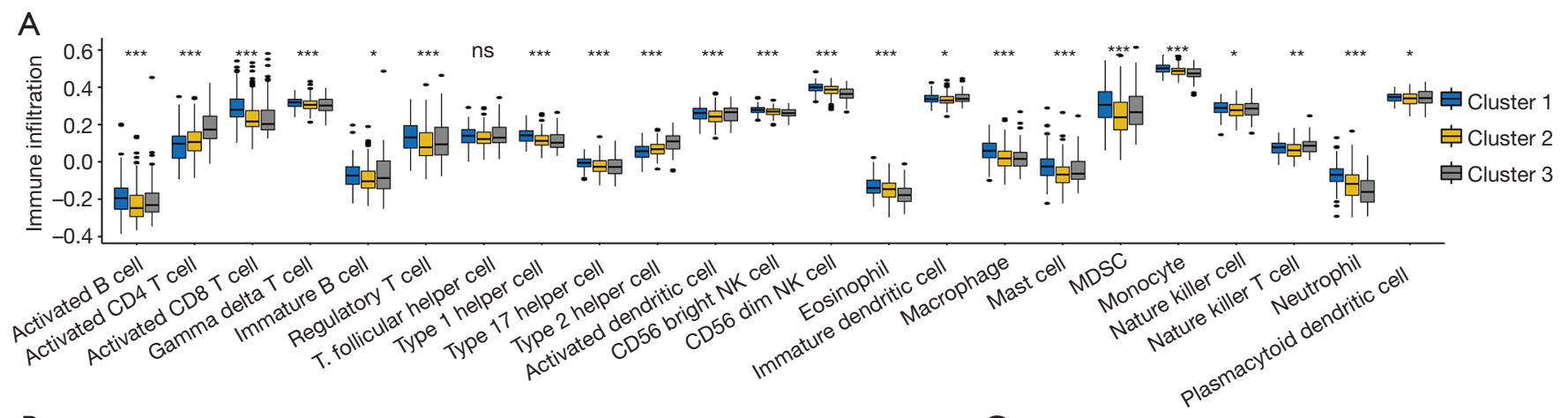

B

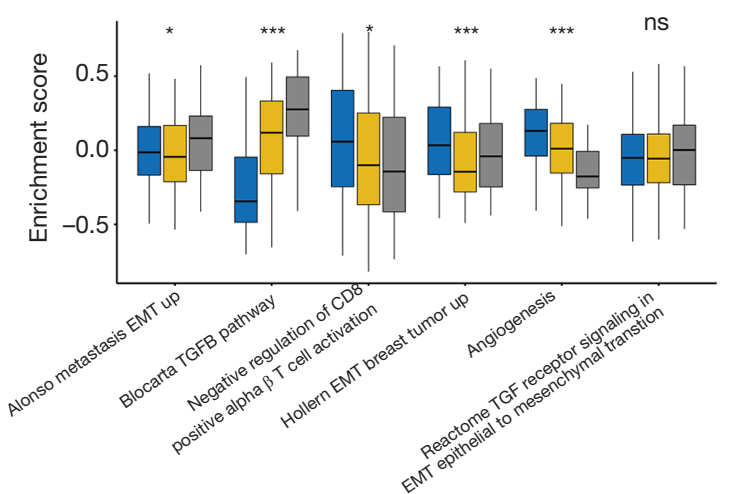

C

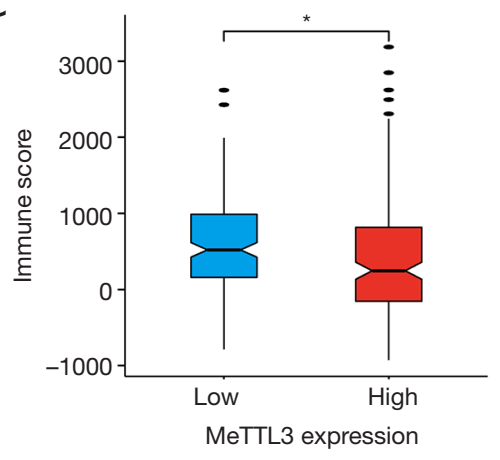

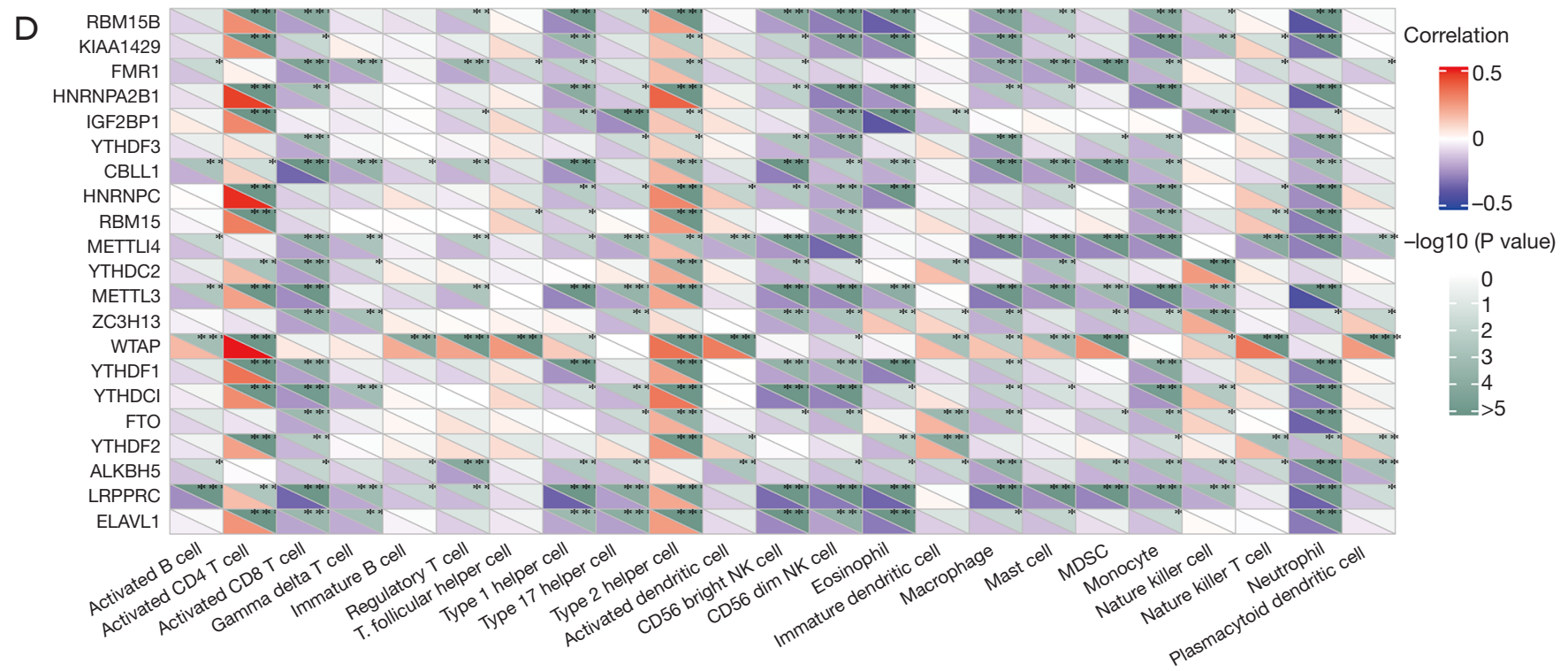

Figure 3 Immune cell infiltration and immune associated classification among m6A regulators. (A) Comparison between different immune cells infiltration in three clusters. (B) Comparison of cellular biological activities with enriched regulation pathways among three clusters. (C) Comparison of the immune score between the high and low METTL3 expression subgroups. The immune score exhibited a weak difference between high and low METTL3 expression levels. (D) Correlation between m6A regulators and biological pathways in the HCC cohort. The Spearman analysis was applied in this study. Negative correlation was marked with blue and positive correlation with orange. ${ }^{*} \mathrm{P}<0.05$, ${ }^{* *} \mathrm{P}<0.01,{ }^{* * *} \mathrm{P}<0.001$. m6A, N6-methyladenosine. 
A

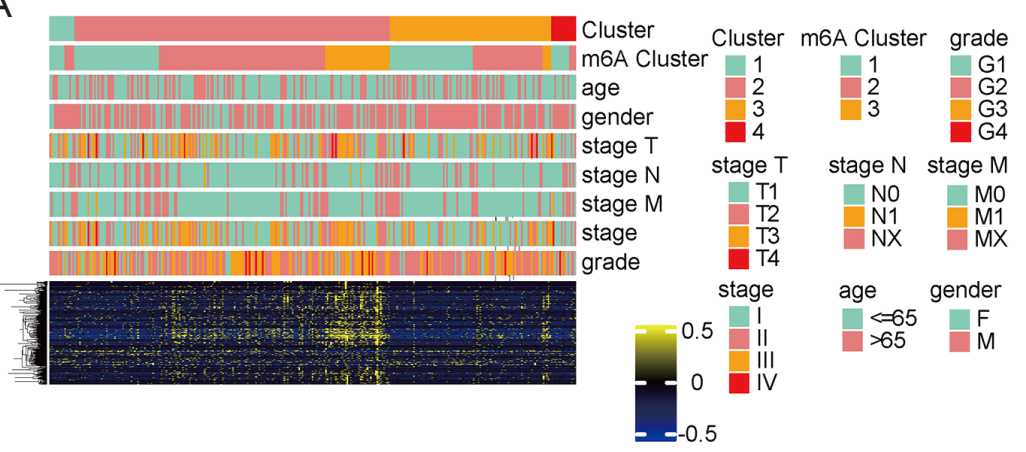

B
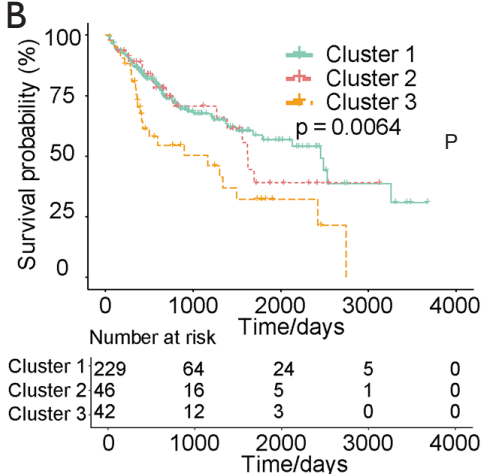
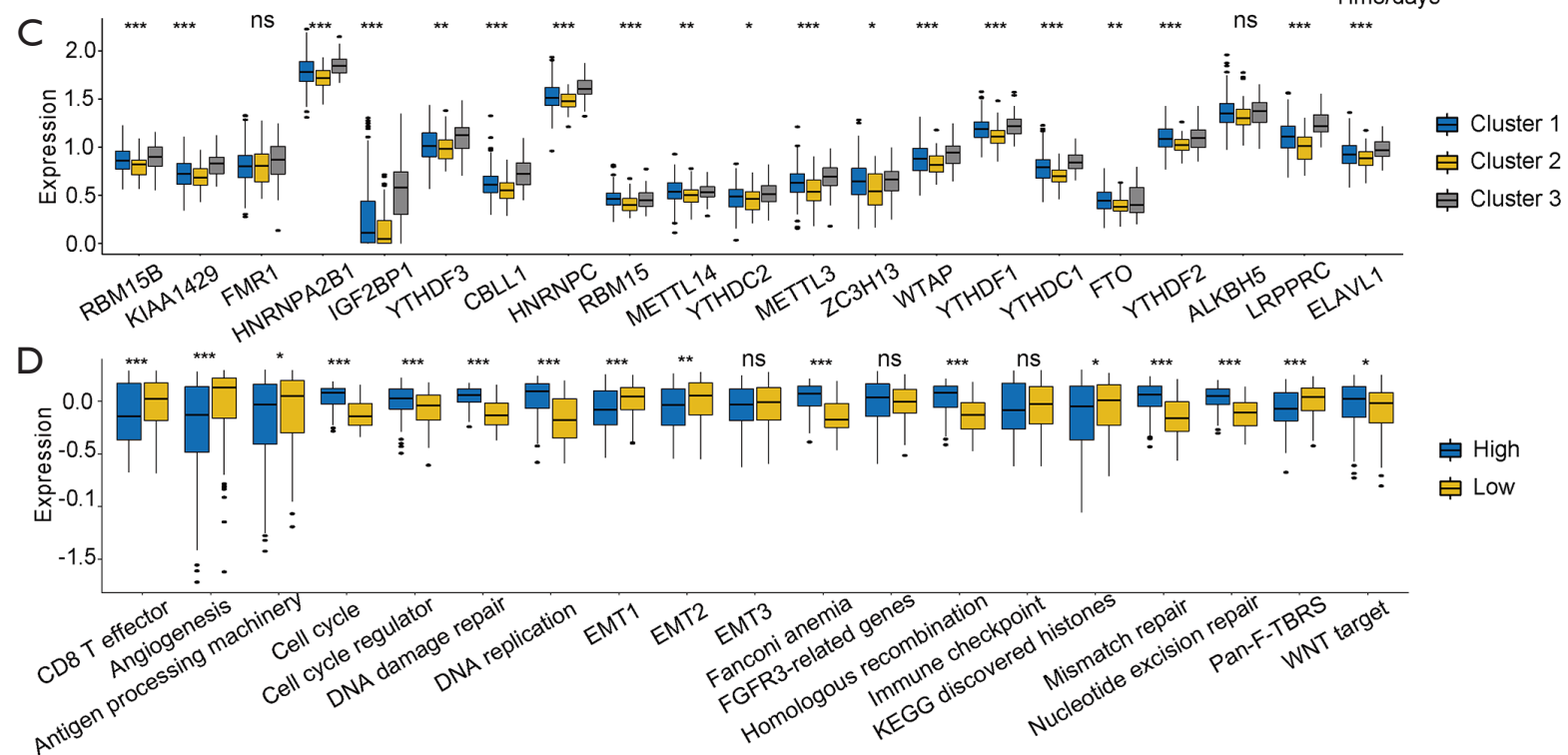

Figure 4 Clinical information in different m6A regulators. (A) m6A regulators were divided into four clusters based on gene expression. Related clinical information was involved in this cluster enrichment analysis. (B) Comparison of overall survival rate between the three different subgroups. (C) Comparison of $\mathrm{m} 6 \mathrm{~A}$ regulators gene expression levels among the three clusters. (D) Comparison of high and low immune score clusters in cellular activities and biological related pathways. m6A, N6-methyladenosine. ${ }^{*} \mathrm{P}<0.05,{ }^{* *} \mathrm{P}<0.01,{ }^{* * *} \mathrm{P}<0.001$.

among the three clusters. Other cytokines exhibited no or weak differences between these clusters (Figure $5 B$ ). In Figure 5C,D, we estimated the gene expression of immune checkpoint molecules and receptors and found that HAVCR2, CD80, PDCD1, TIGIT, CD86, COL4A1, ZEB, TGFB2, and TWIST1 gene expression showed remarkable differences between the three clusters, which suggested that these immune checkpoints had a significant relationship with the $\mathrm{m} 6 \mathrm{~A}$ regulator classification.

We further investigated the prognostic value of the m6A scores for HCC. HCC patients were divided into high and low m6A score groups, and the survival of these groups was compared. The overall survival for the low score m6A group was better than the high score group $[\mathrm{P}=0.019$, HR
$1.6(1.1-2.3)]$. The 5 -year survival rate was $56 \%$ for the high $\mathrm{m} 6 \mathrm{~A}$ score group and $48 \%$ for the low score group (Figure 5E). The area under the curve (AUC) in patients treated with immunotherapy was 0.768 (Figure 5F). Our data demonstrated that the m6A evaluation scores based on modification patterns were correlated with immune or matrix activation, which affects tumor immune infiltrates and patient prognosis.

\section{Discussion}

Globally, HCC is still a lethal malignancy with a poor prognosis. The tumorigenesis mechanism of HCC must be elucidated to improve prognosis, identify biomarkers, 


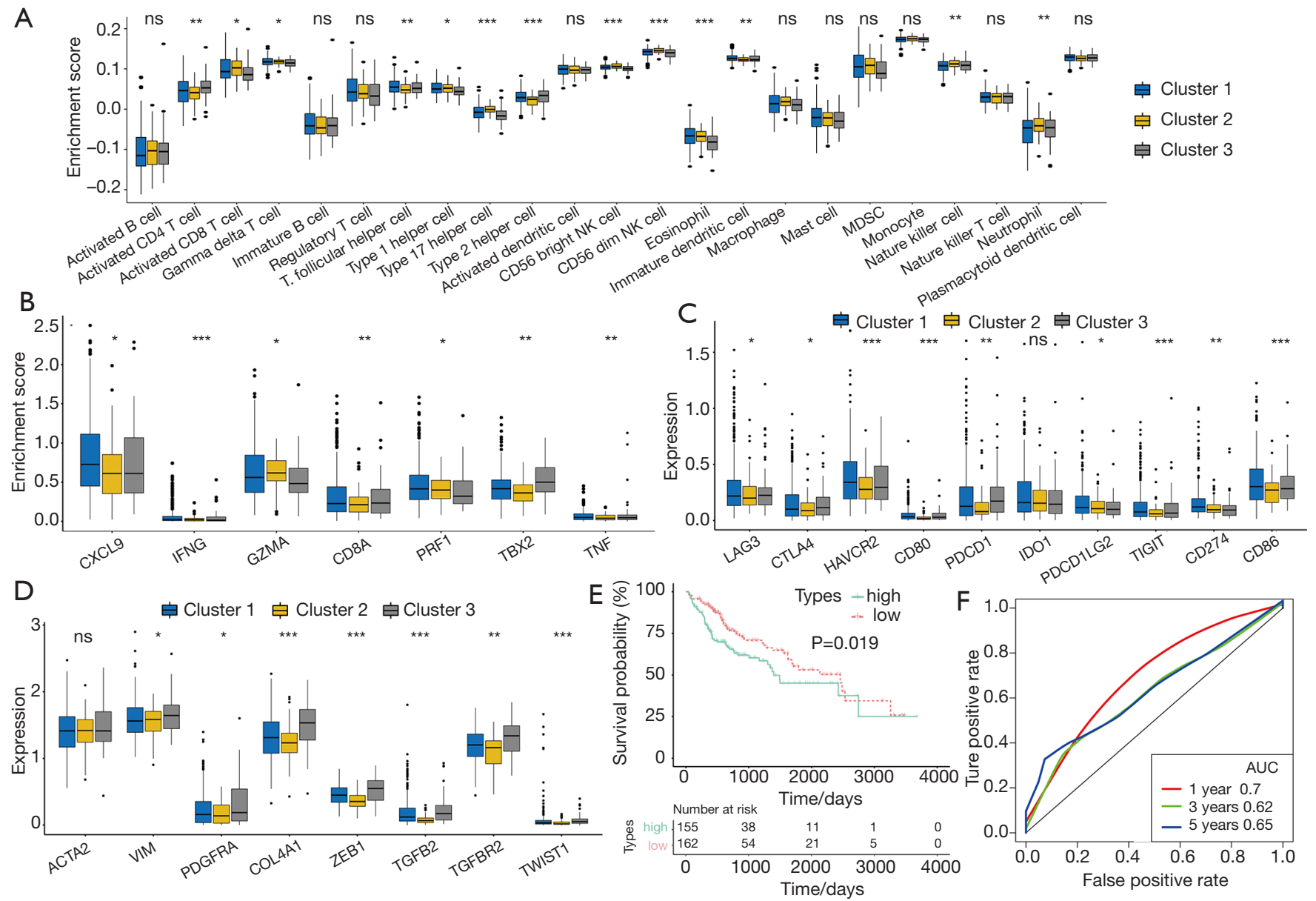

Figure 5 Comparison of different kinds of immune infiltration and prognosis analysis. (A) Comparison of enrichment score with different immune cells infiltration in the three clusters. (B) Comparison of enrichment score with different immune-related cytokines expression among the three clusters. (C) Expression level comparison of different immune checkpoint targets and associated receptors in the three clusters. (D) Expression comparison of m6A regulator-related kinases in the three clusters. (E) Overall survival analysis between the high and low immune score subgroups. (F) AUC of m6A regulators' signature validation of the survival value of the risk score. m6A, N6methyladenosine. ${ }^{*} \mathrm{P}<0.05,{ }^{* *} \mathrm{P}<0.01,{ }^{* *} \mathrm{P}<0.001$.

and develop effective therapeutic strategies. The liver is the largest immune-related organ, and the immune system plays a definitive role in oncogenesis $(20,21)$. Recent studies have indicated that aberrant mRNA modifications contribute to HCC prognosis and overall survival. Currently, m6A is the most well studied post-transcriptional mRNA modification, and evidence suggests that this modification epigenetically affects mRNA processing, translation, and stability (22). Dysregulation of the m6A modification has been shown to be associated with the initiation and progression of various cancers. Thus, exploring the relationship between m6A regulation and the TIME could lead to the design of immune-based targeted therapies for HCC.
METTL3 is a crucial oncogene in several cancers and has been shown to be up-regulated in gastric cancer (23), prostate carcinoma (24), and osteosarcoma (25). Also, methyltransferase like 14 (METTL14) has been shown to play a crucial role in the RNA epigenetics of colorectal cancer, and thus, has prognostic value (26). It is also been shown that $Y T H D F 2$ suppresses cancer cell migration via m6A-associated modification (26). In the present study, we identified three different m6A modification patterns and their relationship with the infiltration of immune cells, such as NK cells, macrophages, eosinophils, mast cells, myeloid-derived suppressor cells (MDSCs), and plasma cell-like DCs, into the HCC microenvironment. Based on 
the infiltration of immune cells and the immune score, we identified three different immune-associated phenotypes of the HCC tumor environment: immune excluded, immune inflamed, and immune desert. The immune excluded phenotype is characterized by the presence of $\mathrm{T}$ cells at the periphery of cancer nests. In the immune excluded phenotype of bladder cancer, there was no PD-L1 expression and only a few tumor-infiltrating lymphocytes (TILs) were present (27). Mariathasan et al. found that combination therapy with TGF-blocking and anti-PD-L1 antibodies decreased TGF signaling in stromal cells, facilitated concentration of $\mathrm{T}$ cells in the centers of tumors, and provoked vigorous anti-tumor immunity and tumor regression (28). Zhao et al. demonstrated that the immune inflamed phenotype of triple-negative breast cancers is characterized by the infiltration of CD8 $+\mathrm{T}$ cells into the tumor parenchyma, indicating that these patients are more likely to benefit from immune checkpoint inhibitor therapy (29). Job et al. reported that the inflamed subtype is characterized by massive $\mathrm{T}$ lymphocyte infiltration as well as activation and upregulation of inflammatory and immune checkpoint pathways. This phenotype is associated with a prolonged survival prognosis (30). Thus, patients with the immune inflamed phenotype may benefit from immunotherapy. In this study, we found that the immune desert phenotype is characterized by CD 8 cell deficiency and is associated with a poor prognosis.

The TIME can predict patient survival in most cancer types, and m6A modification has been shown to affect cell infiltration in the TIME and the epigenetic regulation of the immune response in gastric cancer. M6A has also been shown to regulate the innate immune response by targeting type I interferons $(18,31)$. In the innate immune system, neoantigen-specific immunity is regulated by mRNA methylation via the m6A-binding protein $Y T H D F 1$ (32). In this study, we explored m6A regulatory factor-mediated TIME cell infiltration and found more DCs, including activated DCs, immature DCs, and plasma cell-like DCs in tumors with low METTL3 expression, indicating that METTL3-mediated m6A methylation modification activates DCs in the TIME, thereby enhancing the anti-tumor immune response.

\section{Conclusions}

Our findings demonstrated that numerous immune-related genes regulated by $\mathrm{m} 6 \mathrm{~A}$ modification contribute to the immune phenotype. Moreover, we validated a novel model of m6A modification and immune-related gene regulation to predict HCC prognosis. The immune score and immune classification systems may lead to novel therapeutic targets for HCC.

\section{Acknowledgments}

We thank the patients and investigators who participated in TCGA and the GEO for providing the data analyzed in this study.

Funding: This study was supported by funds from the National Natural Science Foundation of China (No 81902832); the Science and Technology Research Project of Henan Province (No 192102310117 and 202102310115); and the Gandan Xiangzhao Research Fund (No GDXZ2019001 and GDXZ2019007).

\section{Footnote}

Reporting Checklist: The authors have completed the REMARK reporting checklist. Available at http://dx.doi. org/10.21037/atm-20-7396

Conflicts of Interest: All authors have completed the ICMJE uniform disclosure form (available at http://dx.doi. org/10.21037/atm-20-7396). The authors have no conflicts of interest to declare.

Ethical Statement: The authors are accountable for all aspects of the work in ensuring that questions related to the accuracy or integrity of any part of the work are appropriately investigated and resolved. The study was conducted in accordance with the Declaration of Helsinki (as revised in 2013).

Open Access Statement: This is an Open Access article distributed in accordance with the Creative Commons Attribution-NonCommercial-NoDerivs 4.0 International License (CC BY-NC-ND 4.0), which permits the noncommercial replication and distribution of the article with the strict proviso that no changes or edits are made and the original work is properly cited (including links to both the formal publication through the relevant DOI and the license). See: https://creativecommons.org/licenses/by-nc-nd/4.0/.

\section{References}

1. Kempinska K, Malik B, Borkin D, et al. Pharmacologic 
Inhibition of the Menin-MLL Interaction Leads to Transcriptional Repression of PEG10 and Blocks Hepatocellular Carcinoma. Mol Cancer Ther 2018;17:26-38.

2. Llovet JM, Zucman-Rossi J, Pikarsky E, et al. Hepatocellular carcinoma. Nat Rev Dis Primers 2016;2:16018.

3. Yang JD, Hainaut P, Gores GJ, et al. A global view of hepatocellular carcinoma: trends, risk, prevention and management. Nat Rev Gastroenterol Hepatol 2019;16:589-604.

4. Xie QL, Liu Y, Zhu Y. Chromosome region maintenance 1 expression and its association with clinical pathological features in primary carcinoma of the liver. Exp Ther Med 2016;12:59-68.

5. Ou Q, Yu Y, Li A, et al. Association of survival and genomic mutation signature with immunotherapy in patients with hepatocellular carcinoma. Ann Transl Med. 2020;8:230.

6. Gajewski TF, Schreiber H, Fu YX. Innate and adaptive immune cells in the tumor microenvironment. Nat Immunol 2013;14:1014-22.

7. Azambuja JH, Ludwig N, Braganhol E, et al. Inhibition of the Adenosinergic Pathway in Cancer Rejuvenates Innate and Adaptive Immunity. Int J Mol Sci 2019;20:5698.

8. Carone C, Olivani A, Dalla Valle R, et al. Immune Gene Expression Profile in Hepatocellular Carcinoma and Surrounding Tissue Predicts Time to Tumor Recurrence. Liver Cancer 2018;7:277-94.

9. Zhang FP, Huang YP, Luo WX, et al. Construction of a risk score prognosis model based on hepatocellular carcinoma microenvironment. World J Gastroenterol 2020;26:134-53.

10. Hsiao YW, Chiu LT, Chen CH, et al. Tumor-Infiltrating Leukocyte Composition and Prognostic Power in Hepatitis B- and Hepatitis C-Related Hepatocellular Carcinomas. Genes (Basel) 2019;10:630.

11. Zhao X, Chen Y, Mao Q, et al. Overexpression of YTHDF1 is associated with poor prognosis in patients with hepatocellular carcinoma. Cancer Biomark 2018;21:859-68.

12. Han M, Yang G, Lin Q, et al. Determination of Endogenous Bufalin in Serum of Patients With Hepatocellular Carcinoma Based on HPLC-MS/MS. Front Oncol 2020;9:1572.

13. Yang Z, Li J, Feng G, et al. MicroRNA-145 Modulates N6Methyladenosine Levels by Targeting the 3'-Untranslated mRNA Region of the N6-Methyladenosine Binding Y'TH
Domain Family 2 Protein. J Biol Chem 2017;292:3614-23.

14. Colaprico A, Silva TC, Olsen C, et al. TCGAbiolinks: an $\mathrm{R} /$ Bioconductor package for integrative analysis of TCGA data. Nucleic Acids Res 2016;44:e71.

15. Grossman RL. Data Lakes, Clouds, and Commons: A Review of Platforms for Analyzing and Sharing Genomic Data. Trends Genet 2019;35:223-34.

16. Wilkerson MD, Hayes DN. ConsensusClusterPlus: a class discovery tool with confidence assessments and item tracking. Bioinformatics 2010;26:1572-3.

17. Charoentong P, Finotello F, Angelova M, et al. Pancancer Immunogenomic Analyses Reveal GenotypeImmunophenotype Relationships and Predictors of Response to Checkpoint Blockade. Cell Rep 2017;18:248-62.

18. Zhang B, Wu Q, Li B, et al. m6A regulator-mediated methylation modification patterns and tumor microenvironment infiltration characterization in gastric cancer. Mol Cancer 2020;19:53.

19. Qin L, Min S, Shu L, et al. Genetic analysis of N6methyladenosine modification genes in Parkinson's disease. Neurobiol Aging 2020;93:143.e9-143.e13.

20. Angell H, Galon J. From the immune contexture to the Immunoscore: the role of prognostic and predictive immune markers in cancer. Curr Opin Immunol 2013;25:261-7.

21. Tang $X$, Shu Z, Zhang $W$, et al. Clinical significance of the immune cell landscape in hepatocellular carcinoma patients with different degrees of fibrosis. Ann Transl Med 2019;7:528.

22. Zhao BS, Wang X, Beadell AV, et al. m6A-dependent maternal mRNA clearance facilitates zebrafish maternalto-zygotic transition. Nature 2017;542:475-8.

23. Guan K, Liu X, Li J, et al. Expression Status And Prognostic Value Of M6A-associated Genes in Gastric Cancer. J Cancer 2020;11:3027-40.

24. Yuan Y, Du Y, Wang L, Liu X. The M6A methyltransferase METTL3 promotes the development and progression of prostate carcinoma via mediating MYC methylation. J Cancer 2020;11:3588-95.

25. Ling Z, Chen L, Zhao J. m6A-dependent up-regulation of DRG1 by METTL3 and ELAVL1 promotes growth, migration, and colony formation in osteosarcoma. Biosci Rep 2020;40:BSR20200282.

26. Yang X, Zhang S, He C, et al. METTL14 suppresses proliferation and metastasis of colorectal cancer by downregulating oncogenic long non-coding RNA XIST. Mol Cancer 2020;19:46. 
27. Mandelkow T, Blessin NC, Lueerss E, et al. Immune Exclusion Is Frequent in Small-Cell Carcinoma of the Bladder. Dis Markers 2019;2019:2532518.

28. Mariathasan S, Turley SJ, Nickles D, et al. TGF $\beta$ attenuates tumour response to PD-L1 blockade by contributing to exclusion of T cells. Nature 2018;554:544-8.

29. Zhao S, Ma D, Xiao Y, et al. Molecular Subtyping of Triple-Negative Breast Cancers by Immunohistochemistry: Molecular Basis and Clinical Relevance. Oncologist 2020;25:e1481-91.

Cite this article as: Shen S, Yan J, Zhang Y, Dong Z, Xing J, He Y. N6-methyladenosine (m6A)-mediated messenger RNA signatures and the tumor immune microenvironment can predict the prognosis of hepatocellular carcinoma. Ann Transl Med 2021;9(1):59. doi: 10.21037/atm-20-7396
30. Job S, Rapoud D, Dos Santos A, et al. Identification of four immune subtypes characterized by distinct composition and functions of tumor microenvironment in intrahepatic cholangiocarcinoma. Hepatology 2019;72:965-81.

31. Winkler R, Gillis E, Lasman L, et al. m6A modification controls the innate immune response to infection by targeting type I interferons. Nat Immunol 2019;20:173-82.

32. Han D, Liu J, Chen C, et al. Anti-tumour immunity controlled through mRNA m6A methylation and YTHDF1 in dendritic cells. Nature 2019;566:270-4. 


\section{Supplementary}

A

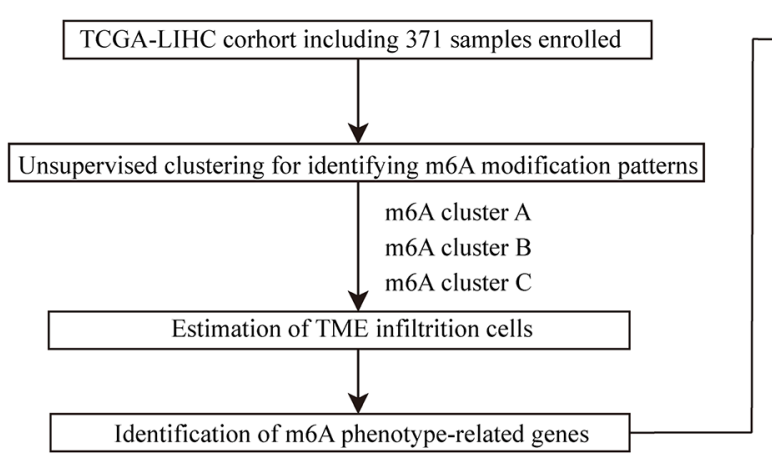

Consensus clustering algorithm for m6A phenotype-related genes

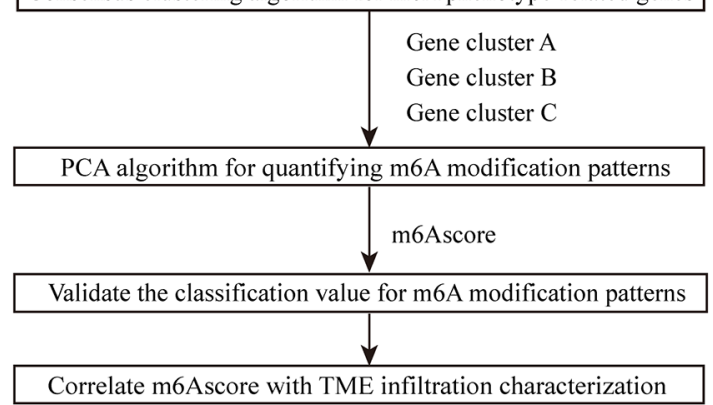

B

all



C

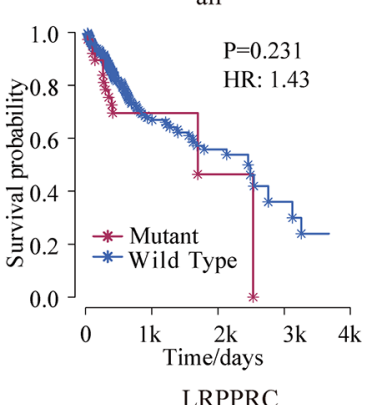

FTO
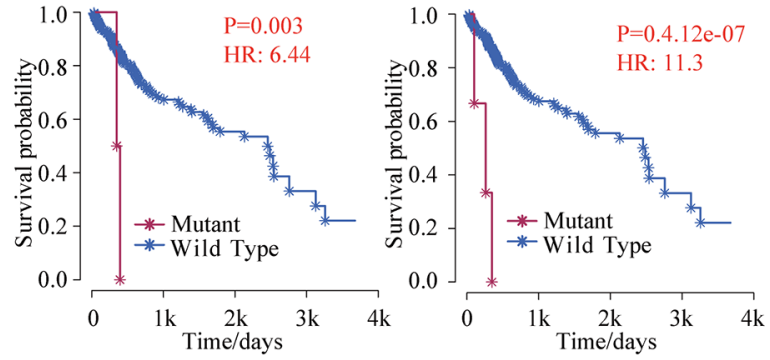

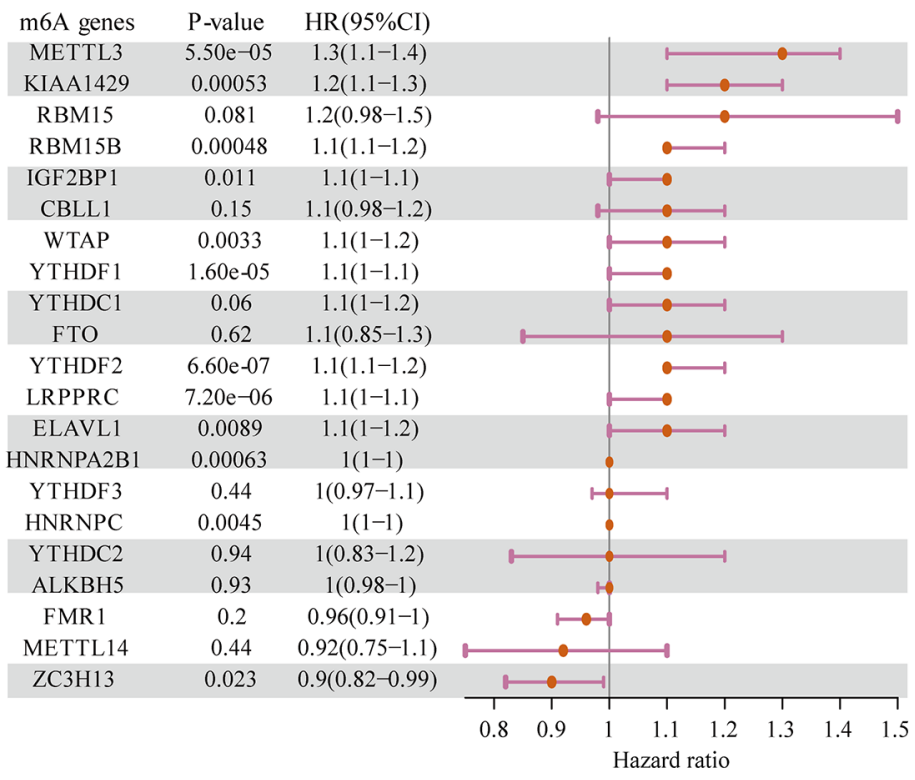

Figure S1 Work flow and survival analysis of m6A regulators. (A) Flow diagram of the analysis procedure, including data collection, preprocessing, and analysis. (B) Overall survival analysis and clinicopathological features between mutated cohort and wide type cohort of regulators. Kaplan-Meier curves of all regulators, YTHDF1, LRPPRC, and FTO. The red represents mutant regulators. (C) Forest plot showing different gene expression of m6A regulators and overall patient survival. m6A, N6-methyladenosine. 

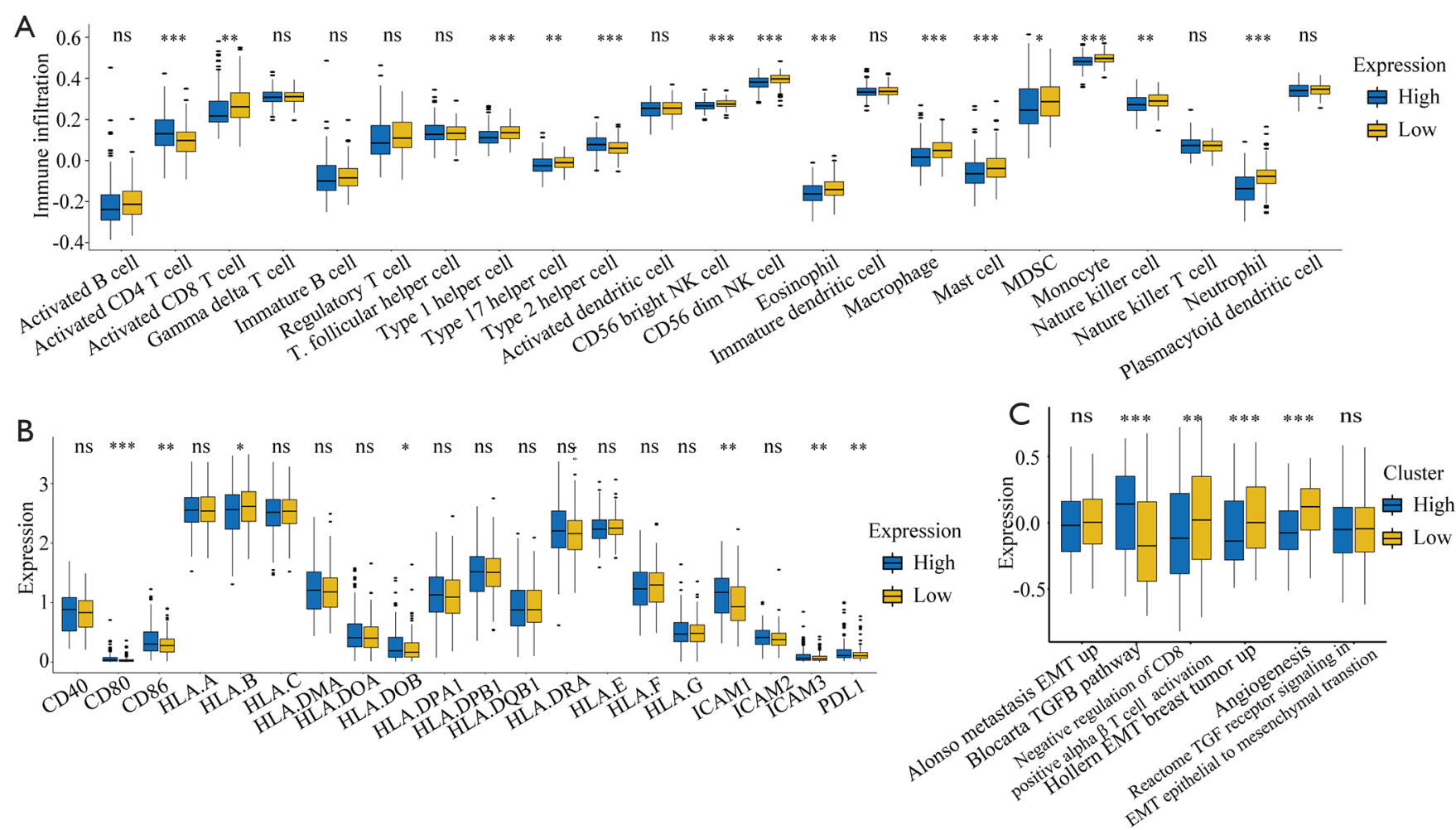

Figure S2 Different m6A regulator expression levels showed different immune cells infiltration. (A) Different METTL3 expression levels had characteristic immune cell infiltration. (B) Different METTL3 expression levels exhibited different immune cell infiltration. (C) Different METTL3 expression levels showed different enriched biological pathways. m6A, N6-methyladenosine; METTL3, methyltransferase like 3. ${ }^{*} \mathrm{P}<0.05,{ }^{* *} \mathrm{P}<0.01,{ }^{* * *} \mathrm{P}<0.001$. 
Table S1 The gene sets used in this work for marking each TME infiltration cell type

\begin{tabular}{|c|c|c|}
\hline Metagene & Cell.type & Immunit \\
\hline ADAM28 & Activated.B.cell & Adaptive \\
\hline CD180 & Activated.B.cell & Adaptive \\
\hline CD79B & Activated.B.cell & Adaptive \\
\hline BLK & Activated.B.cell & Adaptive \\
\hline CD19 & Activated.B.cell & Adaptive \\
\hline MS4A1 & Activated.B.cell & Adaptive \\
\hline TNFRSF17 & Activated.B.cell & Adaptive \\
\hline IGHM & Activated.B.cell & Adaptive \\
\hline GNG7 & Activated.B.cell & Adaptive \\
\hline MICAL3 & Activated.B.cell & Adaptive \\
\hline SPIB & Activated.B.cell & Adaptive \\
\hline HLA-DOB & Activated.B.cell & Adaptive \\
\hline IGKC & Activated.B.cell & Adaptive \\
\hline PNOC & Activated.B.cell & Adaptive \\
\hline FCRL2 & Activated.B.cell & Adaptive \\
\hline BACH2 & Activated.B.cell & Adaptive \\
\hline CR2 & Activated.B.cell & Adaptive \\
\hline TCL1A & Activated.B.cell & Adaptive \\
\hline AKNA & Activated.B.cell & Adaptive \\
\hline ARHGAP25 & Activated.B.cell & Adaptive \\
\hline CCL21 & Activated.B.cell & Adaptive \\
\hline CD27 & Activated.B.cell & Adaptive \\
\hline CD38 & Activated.B.cell & Adaptive \\
\hline CLEC17A & Activated.B.cell & Adaptive \\
\hline CLEC9A & Activated.B.cell & Adaptive \\
\hline CLECL1 & Activated.B.cell & Adaptive \\
\hline AIM2 & Activated.CD4.T.cell & Adaptive \\
\hline BIRC3 & Activated.CD4.T.cell & Adaptive \\
\hline BRIP1 & Activated.CD4.T.cell & Adaptive \\
\hline CCL20 & Activated.CD4.T.cell & Adaptive \\
\hline CCL4 & Activated.CD4.T.cell & Adaptive \\
\hline CCL5 & Activated.CD4.T.cell & Adaptive \\
\hline CCNB1 & Activated.CD4.T.cell & Adaptive \\
\hline CCR7 & Activated.CD4.T.cell & Adaptive \\
\hline DUSP2 & Activated.CD4.T.cell & Adaptive \\
\hline $\mathrm{ESCO} 2$ & Activated.CD4.T.cell & Adaptive \\
\hline ETS1 & Activated.CD4.T.cell & Adaptive \\
\hline EXO1 & Activated.CD4.T.cell & Adaptive \\
\hline EXOC6 & Activated.CD4.T.cell & Adaptive \\
\hline IARS & Activated.CD4.T.cell & Adaptive \\
\hline ITK & Activated.CD4.T.cell & Adaptive \\
\hline KIF11 & Activated.CD4.T.cell & Adaptive \\
\hline KNTC1 & Activated.CD4.T.cell & Adaptive \\
\hline NUF2 & Activated.CD4.T.cell & Adaptive \\
\hline PRC1 & Activated.CD4.T.cell & Adaptive \\
\hline PSAT1 & Activated.CD4.T.cell & Adaptive \\
\hline RGS1 & Activated.CD4.T.cell & Adaptive \\
\hline RTKN2 & Activated.CD4.T.cell & Adaptive \\
\hline SAMSN1 & Activated.CD4.T.cell & Adaptive \\
\hline SELL & Activated.CD4.T.cell & Adaptive \\
\hline TRAT1 & Activated.CD4.T.cell & Adaptive \\
\hline
\end{tabular}




\begin{tabular}{|c|c|c|}
\hline ADRM1 & Activated.CD8.T.cell & Adaptive \\
\hline AHSA1 & Activated.CD8.T.cell & Adaptive \\
\hline C1GALT1C1 & Activated.CD8.T.cell & Adaptive \\
\hline ССТ6В & Activated.CD8.T.cell & Adaptive \\
\hline $\mathrm{CD} 37$ & Activated.CD8.T.cell & Adaptive \\
\hline CD3D & Activated.CD8.T.cell & Adaptive \\
\hline $\mathrm{CD} 3 \mathrm{E}$ & Activated.CD8.T.cell & Adaptive \\
\hline $\mathrm{CD} 3 \mathrm{G}$ & Activated.CD8.T.cell & Adaptive \\
\hline CD69 & Activated.CD8.T.cell & Adaptive \\
\hline CD8A & Activated.CD8.T.cell & Adaptive \\
\hline CETN3 & Activated.CD8.T.cell & Adaptive \\
\hline CSE1L & Activated.CD8.T.cell & Adaptive \\
\hline GEMIN6 & Activated.CD8.T.cell & Adaptive \\
\hline GNLY & Activated.CD8.T.cell & Adaptive \\
\hline GPT2 & Activated.CD8.T.cell & Adaptive \\
\hline GZMA & Activated.CD8.T.cell & Adaptive \\
\hline GZMH & Activated.CD8.T.cell & Adaptive \\
\hline GZMK & Activated.CD8.T.cell & Adaptive \\
\hline IL2RB & Activated.CD8.T.cell & Adaptive \\
\hline LCK & Activated.CD8.T.cell & Adaptive \\
\hline MPZL1 & Activated.CD8.T.cell & Adaptive \\
\hline NKG7 & Activated.CD8.T.cell & Adaptive \\
\hline PIK3IP1 & Activated.CD8.T.cell & Adaptive \\
\hline PTRH2 & Activated.CD8.T.cell & Adaptive \\
\hline TIMM13 & Activated.CD8.T.cell & Adaptive \\
\hline ZAP70 & Activated.CD8.T.cell & Adaptive \\
\hline ACP5 & Gamma.delta.T.cell & Adaptive \\
\hline AQP9 & Gamma.delta.T.cell & Adaptive \\
\hline BTN3A2 & Gamma.delta.T.cell & Adaptive \\
\hline C1orf54 & Gamma.delta.T.cell & Adaptive \\
\hline CARD8 & Gamma.delta.T.cell & Adaptive \\
\hline CCL18 & Gamma.delta.T.cell & Adaptive \\
\hline CD209 & Gamma.delta.T.cell & Adaptive \\
\hline CD33 & Gamma.delta.T.cell & Adaptive \\
\hline CD36 & Gamma.delta.T.cell & Adaptive \\
\hline CDK5 & Gamma.delta.T.cell & Adaptive \\
\hline IL10RB & Gamma.delta.T.cell & Adaptive \\
\hline KLRF1 & Gamma.delta.T.cell & Adaptive \\
\hline LGALS1 & Gamma.delta.T.cell & Adaptive \\
\hline MAPK7 & Gamma.delta.T.cell & Adaptive \\
\hline KLHL7 & Gamma.delta.T.cell & Adaptive \\
\hline KRT80 & Gamma.delta.T.cell & Adaptive \\
\hline LAMC1 & Gamma.delta.T.cell & Adaptive \\
\hline LCORL & Gamma.delta.T.cell & Adaptive \\
\hline LMNB1 & Gamma.delta.T.cell & Adaptive \\
\hline MEIS3P1 & Gamma.delta.T.cell & Adaptive \\
\hline MPL & Gamma.delta.T.cell & Adaptive \\
\hline FABP1 & Gamma.delta.T.cell & Adaptive \\
\hline FABP5 & Gamma.delta.T.cell & Adaptive \\
\hline FADD & Gamma.delta.T.cell & Adaptive \\
\hline MFAP3L & Gamma.delta.T.cell & Adaptive \\
\hline MINPP1 & Gamma.delta.T.cell & Adaptive \\
\hline RPS24 & Gamma.delta.T.cell & Adaptive \\
\hline RPS7 & Gamma.delta.T.cell & Adaptive \\
\hline
\end{tabular}




\begin{tabular}{|c|c|c|}
\hline RPS9 & Gamma.delta.T.cell & Adaptive \\
\hline DBNL & Gamma.delta.T.cell & Adaptive \\
\hline CCL13 & Gamma.delta.T.cell & Adaptive \\
\hline $\mathrm{CD} 22$ & Immature..B.cell & Adaptive \\
\hline CYBB & Immature..B.cell & Adaptive \\
\hline FAM129C & Immature..B.cell & Adaptive \\
\hline FCRL1 & Immature..B.cell & Adaptive \\
\hline FCRL3 & Immature..B.cell & Adaptive \\
\hline FCRL5 & Immature..B.cell & Adaptive \\
\hline FCRLA & Immature..B.cell & Adaptive \\
\hline HDAC9 & Immature..B.cell & Adaptive \\
\hline HLA-DQA1 & Immature..B.cell & Adaptive \\
\hline HVCN1 & Immature..B.cell & Adaptive \\
\hline KIAA0226 & Immature..B.cell & Adaptive \\
\hline NCF1 & Immature..B.cell & Adaptive \\
\hline NCF1B & Immature..B.cell & Adaptive \\
\hline P2RY10 & Immature..B.cell & Adaptive \\
\hline SP100 & Immature..B.cell & Adaptive \\
\hline TXNIP & Immature..B.cell & Adaptive \\
\hline STAP1 & Immature..B.cell & Adaptive \\
\hline TAGAP & Immature..B.cell & Adaptive \\
\hline $\mathrm{ZCCHC} 2$ & Immature..B.cell & Adaptive \\
\hline CCL3L1 & Regulatory.T.cell & Adaptive \\
\hline CD72 & Regulatory.T.cell & Adaptive \\
\hline CLEC5A & Regulatory.T.cell & Adaptive \\
\hline FOXP3 & Regulatory.T.cell & Adaptive \\
\hline ITGA4 & Regulatory.T.cell & Adaptive \\
\hline L1CAM & Regulatory.T.cell & Adaptive \\
\hline LIPA & Regulatory.T.cell & Adaptive \\
\hline LRP1 & Regulatory.T.cell & Adaptive \\
\hline LRRC42 & Regulatory.T.cell & Adaptive \\
\hline MARCO & Regulatory.T.cell & Adaptive \\
\hline MMP12 & Regulatory.T.cell & Adaptive \\
\hline MNDA & Regulatory.T.cell & Adaptive \\
\hline MRC1 & Regulatory.T.cell & Adaptive \\
\hline MS4A6A & Regulatory.T.cell & Adaptive \\
\hline PELO & Regulatory.T.cell & Adaptive \\
\hline PLEK & Regulatory.T.cell & Adaptive \\
\hline PRSS23 & Regulatory.T.cell & Adaptive \\
\hline PTGIR & Regulatory.T.cell & Adaptive \\
\hline ST8SIA4 & Regulatory.T.cell & Adaptive \\
\hline STAB1 & Regulatory.T.cell & Adaptive \\
\hline B3GAT1 & T.follicular.helper.cell & Adaptive \\
\hline CDK5R1 & T.follicular.helper.cell & Adaptive \\
\hline PDCD1 & T.follicular.helper.cell & Adaptive \\
\hline BCL6 & T.follicular.helper.cell & Adaptive \\
\hline CD200 & T.follicular.helper.cell & Adaptive \\
\hline CD83 & T.follicular.helper.cell & Adaptive \\
\hline $\mathrm{CD} 84$ & T.follicular.helper.cell & Adaptive \\
\hline FGF2 & T.follicular.helper.cell & Adaptive \\
\hline GPR18 & T.follicular.helper.cell & Adaptive \\
\hline CEBPA & T.follicular.helper.cell & Adaptive \\
\hline CECR1 & T.follicular.helper.cell & Adaptive \\
\hline CLEC10A & T.follicular.helper.cell & Adaptive \\
\hline
\end{tabular}




\begin{tabular}{|c|c|c|}
\hline CLEC4A & T.follicular.helper.cell & Adaptive \\
\hline CSF1R & T.follicular.helper.cell & Adaptive \\
\hline CTSS & T.follicular.helper.cell & Adaptive \\
\hline DMN & T.follicular.helper.cell & Adaptive \\
\hline DPP4 & T.follicular.helper.cell & Adaptive \\
\hline LRRC32 & T.follicular.helper.cell & Adaptive \\
\hline MC5R & T.follicular.helper.cell & Adaptive \\
\hline MICA & T.follicular.helper.cell & Adaptive \\
\hline NCAM1 & T.follicular.helper.cell & Adaptive \\
\hline NCR2 & T.follicular.helper.cell & Adaptive \\
\hline NRP1 & T.follicular.helper.cell & Adaptive \\
\hline PDCD1LG2 & T.follicular.helper.cell & Adaptive \\
\hline PDCD6 & T.follicular.helper.cell & Adaptive \\
\hline PRDX1 & T.follicular.helper.cell & Adaptive \\
\hline RAE1 & T.follicular.helper.cell & Adaptive \\
\hline RAET1E & T.follicular.helper.cell & Adaptive \\
\hline SIGLEC7 & T.follicular.helper.cell & Adaptive \\
\hline SIGLEC9 & T.follicular.helper.cell & Adaptive \\
\hline TYRO3 & T.follicular.helper.cell & Adaptive \\
\hline CHST12 & T.follicular.helper.cell & Adaptive \\
\hline CLIC3 & T.follicular.helper.cell & Adaptive \\
\hline IVNS1ABP & T.follicular.helper.cell & Adaptive \\
\hline KIR2DL2 & T.follicular.helper.cell & Adaptive \\
\hline LGMN & T.follicular.helper.cell & Adaptive \\
\hline CD70 & Type.1.T.helper.cell & Adaptive \\
\hline TBX21 & Type.1.T.helper.cell & Adaptive \\
\hline ADAM8 & Type.1.T.helper.cell & Adaptive \\
\hline AHCYL2 & Type.1.T.helper.cell & Adaptive \\
\hline ALCAM & Type.1.T.helper.cell & Adaptive \\
\hline B3GALNT1 & Type.1.T.helper.cell & Adaptive \\
\hline BBS12 & Type.1.T.helper.cell & Adaptive \\
\hline BST1 & Type.1.T.helper.cell & Adaptive \\
\hline CD151 & Type.1.T.helper.cell & Adaptive \\
\hline CD47 & Type.1.T.helper.cell & Adaptive \\
\hline CD48 & Type.1.T.helper.cell & Adaptive \\
\hline CD52 & Type.1.T.helper.cell & Adaptive \\
\hline CD53 & Type.1.T.helper.cell & Adaptive \\
\hline CD59 & Type.1.T.helper.cell & Adaptive \\
\hline CD6 & Type.1.T.helper.cell & Adaptive \\
\hline CD68 & Type.1.T.helper.cell & Adaptive \\
\hline CD7 & Type.1.T.helper.cell & Adaptive \\
\hline CD96 & Type.1.T.helper.cell & Adaptive \\
\hline CFHR3 & Type.1.T.helper.cell & Adaptive \\
\hline CHRM3 & Type.1.T.helper.cell & Adaptive \\
\hline CLEC7A & Type.1.T.helper.cell & Adaptive \\
\hline COL23A1 & Type.1.T.helper.cell & Adaptive \\
\hline COL4A4 & Type.1.T.helper.cell & Adaptive \\
\hline COL5A3 & Type.1.T.helper.cell & Adaptive \\
\hline DAB1 & Type.1.T.helper.cell & Adaptive \\
\hline DLEU7 & Type.1.T.helper.cell & Adaptive \\
\hline $\mathrm{DOC} 2 \mathrm{~B}$ & Type.1.T.helper.cell & Adaptive \\
\hline EMP1 & Type.1.T.helper.cell & Adaptive \\
\hline F12 & Type.1.T.helper.cell & Adaptive \\
\hline FURIN & Type.1.T.helper.cell & Adaptive \\
\hline
\end{tabular}




\begin{tabular}{|c|c|c|}
\hline GAB3 & Type.1.T.helper.cell & Adaptive \\
\hline GATM & Type.1.T.helper.cell & Adaptive \\
\hline GFPT2 & Type.1.T.helper.cell & Adaptive \\
\hline GPR25 & Type.1.T.helper.cell & Adaptive \\
\hline GREM2 & Type.1.T.helper.cell & Adaptive \\
\hline HAVCR1 & Type.1.T.helper.cell & Adaptive \\
\hline HSD11B1 & Type.1.T.helper.cell & Adaptive \\
\hline HUNK & Type.1.T.helper.cell & Adaptive \\
\hline IGF2 & Type.1.T.helper.cell & Adaptive \\
\hline RCSD1 & Type.1.T.helper.cell & Adaptive \\
\hline RYR1 & Type.1.T.helper.cell & Adaptive \\
\hline SAV1 & Type.1.T.helper.cell & Adaptive \\
\hline SELE & Type.1.T.helper.cell & Adaptive \\
\hline SELP & Type.1.T.helper.cell & Adaptive \\
\hline SH3KBP1 & Type.1.T.helper.cell & Adaptive \\
\hline SIT1 & Type.1.T.helper.cell & Adaptive \\
\hline SLC35B3 & Type.1.T.helper.cell & Adaptive \\
\hline SIGLEC10 & Type.1.T.helper.cell & Adaptive \\
\hline SKAP1 & Type.1.T.helper.cell & Adaptive \\
\hline THUMPD2 & Type.1.T.helper.cell & Adaptive \\
\hline TIGIT & Type.1.T.helper.cell & Adaptive \\
\hline ZEB2 & Type.1.T.helper.cell & Adaptive \\
\hline ENC1 & Type.1.T.helper.cell & Adaptive \\
\hline FAM134B & Type.1.T.helper.cell & Adaptive \\
\hline FBXO30 & Type.1.T.helper.cell & Adaptive \\
\hline FCGR2C & Type.1.T.helper.cell & Adaptive \\
\hline STAC & Type.1.T.helper.cell & Adaptive \\
\hline LTC4S & Type.1.T.helper.cell & Adaptive \\
\hline MAN1B1 & Type.1.T.helper.cell & Adaptive \\
\hline MDH1 & Type.1.T.helper.cell & Adaptive \\
\hline MMD & Type.1.T.helper.cell & Adaptive \\
\hline RGS16 & Type.1.T.helper.cell & Adaptive \\
\hline IL12A & Type.1.T.helper.cell & Adaptive \\
\hline P2RX5 & Type.1.T.helper.cell & Adaptive \\
\hline CD97 & Type.1.T.helper.cell & Adaptive \\
\hline ITGB4 & Type.1.T.helper.cell & Adaptive \\
\hline ICAM3 & Type.1.T.helper.cell & Adaptive \\
\hline METRNL & Type.1.T.helper.cell & Adaptive \\
\hline TNFRSF1A & Type.1.T.helper.cell & Adaptive \\
\hline IRF1 & Type.1.T.helper.cell & Adaptive \\
\hline HTR2B & Type.1.T.helper.cell & Adaptive \\
\hline CALD1 & Type.1.T.helper.cell & Adaptive \\
\hline MOCOS & Type.1.T.helper.cell & Adaptive \\
\hline TRAF3IP2 & Type.1.T.helper.cell & Adaptive \\
\hline TLR8 & Type.1.T.helper.cell & Adaptive \\
\hline TRAF1 & Type.1.T.helper.cell & Adaptive \\
\hline DUSP14 & Type.1.T.helper.cell & Adaptive \\
\hline IL17A & Type.17.T.helper.cell & Adaptive \\
\hline IL17RA & Type.17.T.helper.cell & Adaptive \\
\hline $\mathrm{C} 2 \mathrm{CD} 4 \mathrm{~A}$ & Type.17.T.helper.cell & Adaptive \\
\hline $\mathrm{C} 2 \mathrm{CD} 4 \mathrm{~B}$ & Type.17.T.helper.cell & Adaptive \\
\hline $\mathrm{CA} 2$ & Type.17.T.helper.cell & Adaptive \\
\hline CCDC65 & Type.17.T.helper.cell & Adaptive \\
\hline CEACAM3 & Type.17.T.helper.cell & Adaptive \\
\hline
\end{tabular}




\begin{tabular}{|c|c|c|}
\hline IL17C & Type.17.T.helper.cell & Adaptive \\
\hline IL17F & Type.17.T.helper.cell & Adaptive \\
\hline IL17RC & Type.17.T.helper.cell & Adaptive \\
\hline IL17RE & Type.17.T.helper.cell & Adaptive \\
\hline IL23A & Type.17.T.helper.cell & Adaptive \\
\hline ILDR1 & Type.17.T.helper.cell & Adaptive \\
\hline LONRF3 & Type.17.T.helper.cell & Adaptive \\
\hline SH2D6 & Type.17.T.helper.cell & Adaptive \\
\hline TNIP2 & Type.17.T.helper.cell & Adaptive \\
\hline ABCA1 & Type.17.T.helper.cell & Adaptive \\
\hline $\mathrm{ABCB} 1$ & Type.17.T.helper.cell & Adaptive \\
\hline ADAMTS12 & Type.17.T.helper.cell & Adaptive \\
\hline ANK1 & Type.17.T.helper.cell & Adaptive \\
\hline ANKRD22 & Type.17.T.helper.cell & Adaptive \\
\hline B3GALT2 & Type.17.T.helper.cell & Adaptive \\
\hline CAMTA1 & Type.17.T.helper.cell & Adaptive \\
\hline CCR9 & Type.17.T.helper.cell & Adaptive \\
\hline CD40 & Type.17.T.helper.cell & Adaptive \\
\hline GPR44 & Type.17.T.helper.cell & Adaptive \\
\hline IFT80 & Type.17.T.helper.cell & Adaptive \\
\hline ASB2 & Type.2.T.helper.cell & Adaptive \\
\hline CSRP2 & Type.2.T.helper.cell & Adaptive \\
\hline DAPK1 & Type.2.T.helper.cell & Adaptive \\
\hline DLC1 & Type.2.T.helper.cell & Adaptive \\
\hline DNAJC12 & Type.2.T.helper.cell & Adaptive \\
\hline DUSP6 & Type.2.T.helper.cell & Adaptive \\
\hline GNAI1 & Type.2.T.helper.cell & Adaptive \\
\hline LAMP3 & Type.2.T.helper.cell & Adaptive \\
\hline NRP2 & Type.2.T.helper.cell & Adaptive \\
\hline OSBPL1A & Type.2.T.helper.cell & Adaptive \\
\hline PDE4B & Type.2.T.helper.cell & Adaptive \\
\hline PHLDA1 & Type.2.T.helper.cell & Adaptive \\
\hline PLA2G4A & Type.2.T.helper.cell & Adaptive \\
\hline RAB27B & Type.2.T.helper.cell & Adaptive \\
\hline RBMS3 & Type.2.T.helper.cell & Adaptive \\
\hline RNF125 & Type.2.T.helper.cell & Adaptive \\
\hline TMPRSS3 & Type.2.T.helper.cell & Adaptive \\
\hline GATA3 & Type.2.T.helper.cell & Adaptive \\
\hline BIRC5 & Type.2.T.helper.cell & Adaptive \\
\hline $\mathrm{CDC} 25 \mathrm{C}$ & Type.2.T.helper.cell & Adaptive \\
\hline CDC7 & Type.2.T.helper.cell & Adaptive \\
\hline CENPF & Type.2.T.helper.cell & Adaptive \\
\hline CXCR6 & Type.2.T.helper.cell & Adaptive \\
\hline DHFR & Type.2.T.helper.cell & Adaptive \\
\hline EVI5 & Type.2.T.helper.cell & Adaptive \\
\hline GSTA4 & Type.2.T.helper.cell & Adaptive \\
\hline HELLS & Type.2.T.helper.cell & Adaptive \\
\hline IL26 & Type.2.T.helper.cell & Adaptive \\
\hline LAIR2 & Type.2.T.helper.cell & Adaptive \\
\hline $\mathrm{ABCD} 1$ & Activated.dendritic.cell & Innate \\
\hline $\mathrm{C} 1 \mathrm{QC}$ & Activated.dendritic.cell & Innate \\
\hline CAPG & Activated.dendritic.cell & Innate \\
\hline CCL3L3 & Activated.dendritic.cell & Innate \\
\hline CD207 & Activated.dendritic.cell & Innate \\
\hline
\end{tabular}


CD302

ATP5B

ATP5L

ATP6V1A

BCL2L1

C1QB

SNURF

SPCS3

CCNA1

CEACAM8

NOS2

SRA1

TNFRSF6B

TREM1

TREML1

RHOA

SLC25A37

TNFSF14

TREML4

VNN2

XPO6

CLEC4C

TNFAIP2

UBD

ACTR3

RAB1A

SLA

HLA-DQA2

SIGLEC5

SLAMF9

ABAT

C11 orf75

C5orf15

CDHR1

DCAF12

DYNLL1

GPR137B

HCP5

HDGFRP2

KRT86

MLST8

ELMOD3

ENTPD5

FAM119A

FAM179A

CLIC2

COX7A2L

CREB3L4

CSF1

CSNK2A2

CSTA

CSTB

CTPS

CTSD
Activated.dendritic.cell

Activated.dendritic.cell

Activated.dendritic.cell

Activated.dendritic.cell

Activated.dendritic.cell

Activated.dendritic.cell

Activated.dendritic.cell

Activated.dendritic.cell

Activated.dendritic.cell

Activated.dendritic.cell

Activated.dendritic.cell

Activated.dendritic.cell

Activated.dendritic.cell

Activated.dendritic.cell

Activated.dendritic.cell

Activated.dendritic.cell

Activated.dendritic.cell

Activated.dendritic.cell

Activated.dendritic.cell

Activated.dendritic.cell

Activated.dendritic.cell

Activated.dendritic.cell

Activated.dendritic.cell

Activated.dendritic.cell

Activated.dendritic.cell

Activated.dendritic.cell

Activated.dendritic.cell

Activated.dendritic.cell

Activated.dendritic.cell

Innate

Innate

Innate

Innate

Innate

Innate

Innate

Innate

Innate

Innate

Innate

Innate

Innate

Innate

Innate

Innate

Innate

Innate

Innate

Innate

Innate

Innate

Innate

Innate

Innate

Innate

Innate

Innate

Innate

Activated.dendritic.cell Innate CD56bright.natural.killer.ce Innate CD56bright.natural.killer.ce Innate CD56bright.natural.killer.ce Innate CD56bright.natural.killer.ce Innate CD56bright.natural.killer.ce Innate CD56bright.natural.killer.ce Innate CD56bright.natural.killer.ce Innate CD56bright.natural.killer.ce Innate CD56bright.natural.killer.ce Innate CD56bright.natural.killer.ce Innate CD56bright.natural.killer.ce Innate CD56bright.natural.killer.ce Innate CD56bright.natural.killer.ce Innate CD56bright.natural.killer.ce Innate CD56bright.natural.killer.ce Innate CD56bright.natural.killer.ce Innate CD56bright.natural.killer.ce Innate CD56bright.natural.killer.ce Innate CD56bright.natural.killer.ce Innate CD56bright.natural.killer.ce Innate CD56bright.natural.killer.ce Innate CD56bright.natural.killer.ce Innate CD56bright.natural.killer.ce Innate CD56bright.natural.killer.ce Innate 


\begin{tabular}{|c|c|}
\hline FST & CD56bright.natural.killer.ce Innate \\
\hline GATA2 & CD56bright.natural.killer.ce Innate \\
\hline GMPR & CD56bright.natural.killer.ce Innate \\
\hline HDC & CD56bright.natural.killer.ce Innate \\
\hline HEY1 & CD56bright.natural.killer.ce Innate \\
\hline HOXA1 & CD56bright.natural.killer.ce Innate \\
\hline HS2ST1 & CD56bright.natural.killer.ce Innate \\
\hline HS3ST1 & CD56bright.natural.killer.ce Innate \\
\hline BCL11B & CD56bright.natural.killer.ce Innate \\
\hline $\mathrm{CDH} 3$ & CD56bright.natural.killer.ce Innate \\
\hline MYL6B & CD56bright.natural.killer.ce Innate \\
\hline NAA16 & CD56bright.natural.killer.ce Innate \\
\hline ClQA & CD56bright.natural.killer.ce Innate \\
\hline $\mathrm{ClQB}$ & CD56bright.natural.killer.ce Innate \\
\hline CYP27B1 & CD56bright.natural.killer.ce Innate \\
\hline EIF3M & CD56bright.natural.killer.ce Innate \\
\hline CYP27A1 & CD56dim.natural.killer.cell Innate \\
\hline DDX55 & CD56dim.natural.killer.cell Innate \\
\hline DYRK2 & CD56dim.natural.killer.cell Innate \\
\hline RPL37A & CD56dim.natural.killer.cell Innate \\
\hline NOTCH3 & CD56dim.natural.killer.cell Innate \\
\hline AKR7A3 & CD56dim.natural.killer.cell Innate \\
\hline GPRC5C & CD56dim.natural.killer.cell Innate \\
\hline GRIN1 & CD56dim.natural.killer.cell Innate \\
\hline HLA-E & CD56dim.natural.killer.cell Innate \\
\hline PORCN & CD56dim.natural.killer.cell Innate \\
\hline PSMC4 & CD56dim.natural.killer.cell Innate \\
\hline UPP1 & CD56dim.natural.killer.cell Innate \\
\hline IL21R & CD56dim.natural.killer.cell Innate \\
\hline KIR2DS1 & CD56dim.natural.killer.cell Innate \\
\hline KIR2DS2 & CD56dim.natural.killer.cell Innate \\
\hline KIR2DS5 & CD56dim.natural.killer.cell Innate \\
\hline GIPR & Eosinophil \\
\hline KRT18P50 & Eosinophil \\
\hline LRMP & Eosinophil \\
\hline FOSB & Eosinophil \\
\hline RRP12 & Eosinophil \\
\hline GPR183 & Eosinophil \\
\hline NR4A3 & Eosinophil \\
\hline ST3GAL6 & Eosinophil \\
\hline DEPDC5 & Eosinophil \\
\hline PDE6C & Eosinophil \\
\hline PKD2L2 & Eosinophil \\
\hline GPR65 & Eosinophil \\
\hline IL5RA & Eosinophil \\
\hline P2RY14 & Eosinophil \\
\hline DACH1 & Eosinophil \\
\hline DAPK2 & Eosinophil \\
\hline EMR3 & Eosinophil \\
\hline ACADM & Immature.dendritic.cell \\
\hline AHCYL1 & Immature.dendritic.cell \\
\hline ALDH1A2 & Immature.dendritic.cell \\
\hline ALDH3A2 & Immature.dendritic.cell \\
\hline ALDH9A1 & Immature.dendritic.cell \\
\hline
\end{tabular}




\begin{tabular}{|c|c|c|}
\hline ALOX15 & Immature.dendritic.cell & Innate \\
\hline AMT & Immature.dendritic.cell & Innate \\
\hline ARL1 & Immature.dendritic.cell & Innate \\
\hline ATIC & Immature.dendritic.cell & Innate \\
\hline ATP5A1 & Immature.dendritic.cell & Innate \\
\hline CAPZA1 & Immature.dendritic.cell & Innate \\
\hline LILRA5 & Immature.dendritic.cell & Innate \\
\hline RDX & Immature.dendritic.cell & Innate \\
\hline RRAGD & Immature.dendritic.cell & Innate \\
\hline TACSTD2 & Immature.dendritic.cell & Innate \\
\hline INPP5F & Immature.dendritic.cell & Innate \\
\hline RAB38 & Immature.dendritic.cell & Innate \\
\hline PLAU & Immature.dendritic.cell & Innate \\
\hline CSF3R & Immature.dendritic.cell & Innate \\
\hline SLC18A2 & Immature.dendritic.cell & Innate \\
\hline AMPD2 & Immature.dendritic.cell & Innate \\
\hline CLTB & Immature.dendritic.cell & Innate \\
\hline $\mathrm{C} 1$ orf162 & Immature.dendritic.cell & Innate \\
\hline AIF1 & Macrophage & Innate \\
\hline CCL1 & Macrophage & Innate \\
\hline CCL14 & Macrophage & Innate \\
\hline CCL23 & Macrophage & Innate \\
\hline CCL26 & Macrophage & Innate \\
\hline CD300LB & Macrophage & Innate \\
\hline CNR1 & Macrophage & Innate \\
\hline CNR2 & Macrophage & Innate \\
\hline EIF1 & Macrophage & Innate \\
\hline EIF4A1 & Macrophage & Innate \\
\hline FPR1 & Macrophage & Innate \\
\hline FPR2 & Macrophage & Innate \\
\hline FRAT2 & Macrophage & Innate \\
\hline GPR27 & Macrophage & Innate \\
\hline GPR77 & Macrophage & Innate \\
\hline RNASE2 & Macrophage & Innate \\
\hline MS4A2 & Macrophage & Innate \\
\hline BASP1 & Macrophage & Innate \\
\hline IGSF6 & Macrophage & Innate \\
\hline HK3 & Macrophage & Innate \\
\hline VNN1 & Macrophage & Innate \\
\hline FES & Macrophage & Innate \\
\hline NPL & Macrophage & Innate \\
\hline FZD2 & Macrophage & Innate \\
\hline FAM198B & Macrophage & Innate \\
\hline HNMT & Macrophage & Innate \\
\hline SLC15A3 & Macrophage & Innate \\
\hline CD4 & Macrophage & Innate \\
\hline TXNDC3 & Macrophage & Innate \\
\hline FRMD4A & Macrophage & Innate \\
\hline CRYBB1 & Macrophage & Innate \\
\hline HRH1 & Macrophage & Innate \\
\hline WNT5B & Macrophage & Innate \\
\hline ADAMTS3 & Mast.cell & Innate \\
\hline CPA3 & Mast.cell & Innate \\
\hline CMA1 & Mast.cell & Innate \\
\hline
\end{tabular}




\begin{tabular}{|c|c|c|}
\hline CTSG & Mast.cell & Innate \\
\hline ARHGAP15 & Mast.cell & Innate \\
\hline CPM & Mast.cell & Innate \\
\hline FCN1 & Mast.cell & Innate \\
\hline FTL & Mast.cell & Innate \\
\hline HSPA6 & Mast.cell & Innate \\
\hline ITGA9 & Mast.cell & Innate \\
\hline RNASE3 & Mast.cell & Innate \\
\hline S100A4 & Mast.cell & Innate \\
\hline SIGLEC8 & Mast.cell & Innate \\
\hline SLC6A4 & Mast.cell & Innate \\
\hline PTGS2 & Mast.cell & Innate \\
\hline EGR3 & Mast.cell & Innate \\
\hline PILRA & Mast.cell & Innate \\
\hline CCR2 & MDSC & Innate \\
\hline CD14 & MDSC & Innate \\
\hline $\mathrm{CD} 2$ & MDSC & Innate \\
\hline CD86 & MDSC & Innate \\
\hline CXCR4 & MDSC & Innate \\
\hline FCGR2A & MDSC & Innate \\
\hline FCGR2B & MDSC & Innate \\
\hline FCGR3A & MDSC & Innate \\
\hline FERMT3 & MDSC & Innate \\
\hline GPSM3 & MDSC & Innate \\
\hline IL18BP & MDSC & Innate \\
\hline IL4R & MDSC & Innate \\
\hline ITGAL & MDSC & Innate \\
\hline ITGAM & MDSC & Innate \\
\hline PARVG & MDSC & Innate \\
\hline PSAP & MDSC & Innate \\
\hline PTGER2 & MDSC & Innate \\
\hline PTGES2 & MDSC & Innate \\
\hline S100A8 & MDSC & Innate \\
\hline S100A9 & MDSC & Innate \\
\hline ASGR2 & Monocyte & Innate \\
\hline CFP & Monocyte & Innate \\
\hline ASGR1 & Monocyte & Innate \\
\hline CD1D & Monocyte & Innate \\
\hline UPK3A & Monocyte & Innate \\
\hline ACTG1 & Monocyte & Innate \\
\hline ANXA5 & Monocyte & Innate \\
\hline ATP6V1B2 & Monocyte & Innate \\
\hline CFL1 & Monocyte & Innate \\
\hline DAZAP2 & Monocyte & Innate \\
\hline CTBS & Monocyte & Innate \\
\hline EMR4P & Monocyte & Innate \\
\hline HIVEP2 & Monocyte & Innate \\
\hline MARCKSL1 & Monocyte & Innate \\
\hline MBP & Monocyte & Innate \\
\hline MMP15 & Monocyte & Innate \\
\hline PNPLA6 & Monocyte & Innate \\
\hline TMBIM6 & Monocyte & Innate \\
\hline PQBP1 & Monocyte & Innate \\
\hline TEX264 & Monocyte & Innate \\
\hline
\end{tabular}




\begin{tabular}{|c|c|c|}
\hline IKZF1 & Monocyte & Innate \\
\hline AKT3 & Natural.killer.cell & Innate \\
\hline AXL & Natural.killer.cell & Innate \\
\hline BST2 & Natural.killer.cell & Innate \\
\hline $\mathrm{CDH} 2$ & Natural.killer.cell & Innate \\
\hline CRTAM & Natural.killer.cell & Innate \\
\hline CSF2RA & Natural.killer.cell & Innate \\
\hline CTSZ & Natural.killer.cell & Innate \\
\hline CXCL1 & Natural.killer.cell & Innate \\
\hline CYTH1 & Natural.killer.cell & Innate \\
\hline DAXX & Natural.killer.cell & Innate \\
\hline DGKH & Natural.killer.cell & Innate \\
\hline DLL4 & Natural.killer.cell & Innate \\
\hline DPYD & Natural.killer.cell & Innate \\
\hline ERBB3 & Natural.killer.cell & Innate \\
\hline F11R & Natural.killer.cell & Innate \\
\hline FAM27A & Natural.killer.cell & Innate \\
\hline FAM49A & Natural.killer.cell & Innate \\
\hline FASLG & Natural.killer.cell & Innate \\
\hline FCGR1A & Natural.killer.cell & Innate \\
\hline FN1 & Natural.killer.cell & Innate \\
\hline FSTL1 & Natural.killer.cell & Innate \\
\hline FUCA1 & Natural.killer.cell & Innate \\
\hline GBP3 & Natural.killer.cell & Innate \\
\hline GLS2 & Natural.killer.cell & Innate \\
\hline GRB2 & Natural.killer.cell & Innate \\
\hline LST1 & Natural.killer.cell & Innate \\
\hline BCL2 & Natural.killer.cell & Innate \\
\hline CDC5L & Natural.killer.cell & Innate \\
\hline FGF18 & Natural.killer.cell & Innate \\
\hline FUT5 & Natural.killer.cell & Innate \\
\hline FZR1 & Natural.killer.cell & Innate \\
\hline GAGE2 & Natural.killer.cell & Innate \\
\hline IGFBP5 & Natural.killer.cell & Innate \\
\hline KANK2 & Natural.killer.cell & Innate \\
\hline LDB3 & Natural.killer.cell & Innate \\
\hline BTN2A2 & Natural.killer.T.cell & Innate \\
\hline $\mathrm{CD} 101$ & Natural.killer.T.cell & Innate \\
\hline CD109 & Natural.killer.T.cell & Innate \\
\hline CNPY3 & Natural.killer.T.cell & Innate \\
\hline CNPY4 & Natural.killer.T.cell & Innate \\
\hline CREB1 & Natural.killer.T.cell & Innate \\
\hline CRTC2 & Natural.killer.T.cell & Innate \\
\hline CRTC3 & Natural.killer.T.cell & Innate \\
\hline CSF2 & Natural.killer.T.cell & Innate \\
\hline KLRC1 & Natural.killer.T.cell & Innate \\
\hline FUT4 & Natural.killer.T.cell & Innate \\
\hline ICAM2 & Natural.killer.T.cell & Innate \\
\hline IL32 & Natural.killer.T.cell & Innate \\
\hline LAMP2 & Natural.killer.T.cell & Innate \\
\hline LILRB5 & Natural.killer.T.cell & Innate \\
\hline KLRG1 & Natural.killer.T.cell & Innate \\
\hline HSPA4 & Natural.killer.T.cell & Innate \\
\hline HSPB6 & Natural.killer.T.cell & Innat \\
\hline
\end{tabular}




\begin{tabular}{|c|c|c|}
\hline ISM2 & Natural.killer.T.cell & Innate \\
\hline ITIH2 & Natural.killer.T.cell & Innate \\
\hline KDM4C & Natural.killer.T.cell & Innate \\
\hline KIR2DS4 & Natural.killer.T.cell & Innate \\
\hline KIRREL3 & Natural.killer.T.cell & Innate \\
\hline SDCBP & Natural.killer.T.cell & Innate \\
\hline NFATC2IP & Natural.killer.T.cell & Innate \\
\hline MICB & Natural.killer.T.cell & Innate \\
\hline KIR2DL1 & Natural.killer.T.cell & Innate \\
\hline KIR2DL3 & Natural.killer.T.cell & Innate \\
\hline KIR3DL1 & Natural.killer.T.cell & Innate \\
\hline KIR3DL2 & Natural.killer.T.cell & Innate \\
\hline NCR1 & Natural.killer.T.cell & Innate \\
\hline FOSL1 & Natural.killer.T.cell & Innate \\
\hline TSLP & Natural.killer.T.cell & Innate \\
\hline SLC7A7 & Natural.killer.T.cell & Innate \\
\hline SPP1 & Natural.killer.T.cell & Innate \\
\hline TREM2 & Natural.killer.T.cell & Innate \\
\hline UBASH3A & Natural.killer.T.cell & Innate \\
\hline YBX2 & Natural.killer.T.cell & Innate \\
\hline CCDC88A & Natural.killer.T.cell & Innate \\
\hline CLEC1A & Natural.killer.T.cell & Innate \\
\hline THBD & Natural.killer.T.cell & Innate \\
\hline PDPN & Natural.killer.T.cell & Innate \\
\hline VCAM1 & Natural.killer.T.cell & Innate \\
\hline EMR1 & Natural.killer.T.cell & Innate \\
\hline CREB5 & Neutrophil & Innate \\
\hline CDA & Neutrophil & Innate \\
\hline CHST15 & Neutrophil & Innate \\
\hline S100A12 & Neutrophil & Innate \\
\hline APOBEC3A & Neutrophil & Innate \\
\hline CASP5 & Neutrophil & Innate \\
\hline MMP25 & Neutrophil & Innate \\
\hline HAL & Neutrophil & Innate \\
\hline C1orf183 & Neutrophil & Innate \\
\hline FFAR2 & Neutrophil & Innate \\
\hline MAK & Neutrophil & Innate \\
\hline CXCR1 & Neutrophil & Innate \\
\hline STEAP4 & Neutrophil & Innate \\
\hline MGAM & Neutrophil & Innate \\
\hline BTNL8 & Neutrophil & Innate \\
\hline CXCR2 & Neutrophil & Innate \\
\hline TNFRSF10C & Neutrophil & Innate \\
\hline VNN3 & Neutrophil & Innate \\
\hline CBX6 & Plasmacytoid.dendritic.cell & Innate \\
\hline DAB2 & Plasmacytoid.dendritic.cell & Innate \\
\hline DDX17 & Plasmacytoid.dendritic.cell & Innate \\
\hline HIGD1A & Plasmacytoid.dendritic.cell & Innate \\
\hline IDH3A & Plasmacytoid.dendritic.cell & Innate \\
\hline IL3RA & Plasmacytoid.dendritic.cell & Innate \\
\hline MAGED1 & Plasmacytoid.dendritic.cell & Innate \\
\hline NUCB2 & Plasmacytoid.dendritic.cell & Innate \\
\hline OFD1 & Plasmacytoid.dendritic.cell & Innate \\
\hline OGT & Plasmacytoid.dendritic.cell & Innate \\
\hline
\end{tabular}




$\begin{array}{ll}\text { PDIA4 } & \text { Plasmacytoid.dendritic.cell Innate } \\ \text { SERTAD2 } & \text { Plasmacytoid.dendritic.cell Innate } \\ \text { SIRPA } & \text { Plasmacytoid.dendritic.cell Innate } \\ \text { TMED2 } & \text { Plasmacytoid.dendritic.cell Innate } \\ \text { ENG } & \text { Plasmacytoid.dendritic.cell Innate } \\ \text { FCAR } & \text { Plasmacytoid.dendritic.cell Innate } \\ \text { IGF1 } & \text { Plasmacytoid.dendritic.cell Innate } \\ \text { ITGA2B } & \text { Plasmacytoid.dendritic.cell Innate } \\ \text { GABARAP } & \text { Plasmacytoid.dendritic.cell Innate } \\ \text { GPX1 } & \text { Plasmacytoid.dendritic.cell Innate } \\ \text { KRT23 } & \text { Plasmacytoid.dendritic.cell Innate } \\ \text { PROK2 } & \text { Plasmacytoid.dendritic.cell Innate } \\ \text { RALB } & \text { Plasmacytoid.dendritic.cell Innate } \\ \text { RETNLB } & \text { Plasmacytoid.dendritic.cell Innate } \\ \text { RNF141 } & \text { Plasmacytoid.dendritic.cell Innate } \\ \text { SEC14L1 } & \text { Plasmacytoid.dendritic.cell Innate } \\ \text { SEPX1 } & \text { Plasmacytoid.dendritic.cell Innate } \\ \text { EMP3 } & \text { Plasmacytoid.dendritic.cell Innate } \\ \text { CD300LF } & \text { Plasmacytoid.dendritic.cell Innate } \\ \text { ABTB1 } & \text { Plasmacytoid.dendritic.cell Innate } \\ \text { KLHL21 } & \text { Plasmacytoid.dendritic.cell Innate } \\ \text { PHRF1 } & \text { Plasmacytoid.dendritic.cell Innate }\end{array}$

